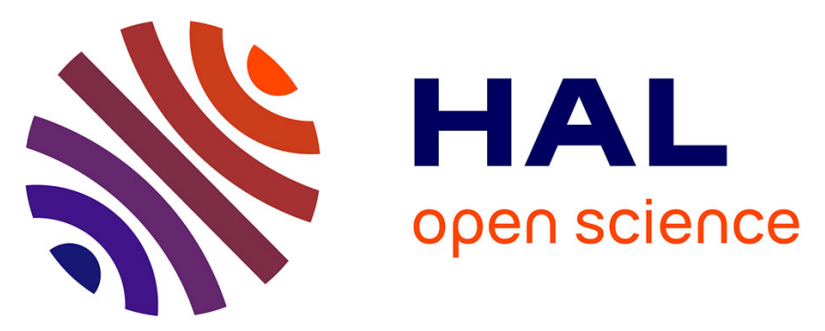

\title{
Wavenumber explicit convergence analysis for finite element discretizations of time-harmonic wave propagation problems with perfectly matched layers
}

Théophile Chaumont-Frelet, Dietmar Gallistl, Serge Nicaise, Jérôme Tomezyk

\section{- To cite this version:}

Théophile Chaumont-Frelet, Dietmar Gallistl, Serge Nicaise, Jérôme Tomezyk. Wavenumber explicit convergence analysis for finite element discretizations of time-harmonic wave propagation problems with perfectly matched layers. Communications in Mathematical Sciences, 2022, 20 (1), pp.1-52. 10.4310/CMS.2022.v20.n1.a1 . hal-01887267

\section{HAL Id: hal-01887267 https://hal.science/hal-01887267}

Submitted on 3 Oct 2018

HAL is a multi-disciplinary open access archive for the deposit and dissemination of scientific research documents, whether they are published or not. The documents may come from teaching and research institutions in France or abroad, or from public or private research centers.
L'archive ouverte pluridisciplinaire HAL, est destinée au dépôt et à la diffusion de documents scientifiques de niveau recherche, publiés ou non, émanant des établissements d'enseignement et de recherche français ou étrangers, des laboratoires publics ou privés. 


\title{
WAVENUMBER EXPLICIT CONVERGENCE ANALYSIS FOR FINITE ELEMENT DISCRETIZATIONS OF TIME-HARMONIC WAVE PROPAGATION PROBLEMS WITH PERFECTLY MATCHED LAYERS
}

\author{
T. CHAUMONT-FRELET, D. GALLISTL, S. NICAISE, AND J. TOMEZYK
}

\begin{abstract}
The first part of this paper is devoted to a wavenumber-explicit stability analysis of a planar Helmholtz problem with a perfectly matched layer. We prove that, for a model scattering problem, the $H^{1}$ norm of the solution is bounded by the righthand side, uniformly in the wavenumber $k$ in the highly oscillatory regime. The second part proposes two numerical discretizations: an $h p$ finite element method and a multiscale method based on local subspace correction. The stability result is used to relate the choice of parameters in the numerical methods to the wavenumber. A priori error estimates are shown and their sharpness is assessed in numerical experiments.
\end{abstract}

\section{INTRODUCTION}

Time harmonic acoustic scattering problems are often modelled through the Helmholtz equation in full space subject to the Sommerfeld radiation condition [10]. The simulation with finite elements requires truncation to a finite domain. An alternative to absorbing boundary conditions, that classically replace the radiation condition in this case, is to introduce a so-called Perfectly Matched Layer (PML) near the boundary, in which a fictitious absorption coefficient avoids artificial reflections. This procedure was introduced in [2] and thereafter studied in many contributions (see, e.g., [9] and the references therein).

The governing elliptic differential operator, the Helmholtz operator $\left(\Delta+k^{2}\right)$, depends on the wavenumber $k$. We are interested in the highly oscillatory regime, which corresponds to large values of $k$. It is known that the finite element method (FEM) is not robust with respect to that parameter [1]. This phenomenon is usually referred to as pollution effect. It essentially states that a fixed number of grid points per wavelength is insufficient for numerical stability, although it would provide a reasonable approximation. The design and the analysis of numerical methods avoiding pollution has been an active area of numerical analysis. The stability of the underlying partial differential equation (PDE) with respect to $k$ is crucial for the analysis of numerical methods. While there are extreme cases [4], in

2010 Mathematics Subject Classification. Primary 35J05 65N12 65N30; Secondary 78A40.

Key words and phrases. Helmholtz problems; PML; High order methods; Finite elements; Multiscale method; Pollution effect.

D. Gallistl acknowledges support by the DFG through SFB 1173 and by the Baden-Württemberg Stiftung through the project "Mehrskalenmethoden für Wellenausbreitung in heterogenen Materialien und Metamaterialien". 
which the stability constant depends exponentially on $k$, for a couple of relevant settings there are results on polynomial stability [12, 14, 17, 22, 24, 25, 28]

We mention two numerical methods that overcome the pollution effect and are relevant to our work. In [23] it is shown that $h p$ finite elements are pollution-free if the polynomial degree is adequately coupled with $k$. A different approach based on fine-scale correction techniques was proposed by [26]. It is based on low-order polynomials, but the diameter of the support of the fine-scale corrections must grow logarithmically with $k$.

In this work we study $h p$ finite elements and a multiscale method for a two-dimensional Helmholtz setting with PML. We derive a stability result that shows that the solution (measured in a scaled $H^{1}$ norm) is robustly bounded by the $L^{2}$ norm of the right-hand side. The proof is based on the combination of a direct estimate obtained in the PML region with a multiplier method (in the case of absorbing boundary conditions this last procedure corresponds to the choice of an appropriate test function, see [22]).

The remaining parts of this paper are organized as follows. Section 2 states the PML setting in polar coordinates. The main stability estimate is proven in Section 3. In Section 4 we study the comparison with a sponge layer, which leads to an appropriate expansion of the solution. The numerical proposed discretizations are described in Section 5 and computational experiments are given Section 6. We conclude with some remarks in Section 7. Appendix A lists a couple of elementary but important properties of the PML functions that we often refer to.

We finish this section with some notations used in the remainder of the paper. For a bounded domain $D$, the usual norm and semi-norm of $H^{s}(D)(s \geq 0)$ are denoted by $\|\cdot\|_{s, D}$ and $|\cdot|_{s, D}$, respectively. For $s=0$, we drop the index 0 for shortness. The space of smooth functions with compact support in $D$ is denoted by $\mathcal{D}(D)$. Furthermore, the notation $A \lesssim B$ (resp. $A \gtrsim B$ ) means the existence of a positive constant $C_{1}$ (resp. $C_{2}$ ), which is independent of $A, B$ as well as on the wavenumber $k$ and the mesh size $h$. such that $A \leq C_{1} B$ (resp. $A \geq C_{2} B$ ). The notation $A \sim B$ means that $A \lesssim B$ and $A \gtrsim B$ hold simultaneously.

\section{The SCATtering PROBLEM With A POLAR PML}

We consider the Helmholtz equation set in the outside of a smooth, star-shaped soundsoft obstacle $\mathcal{O} \subset \mathbb{R}^{2}$. In order to approximate this problem with finite elements, it is required to truncate the computational domain. Here, we propose to analyze the Helmholtz equation when a Perfectly Matched Layer (PML) is employed.

Without losing generality, we select the coordinate system so that $\mathcal{O}$ is star-shaped with respect to the origin. We introduce two positive numbers $a<b$ such that $\mathcal{O}$ is contained in $B(0, a)$, the ball of $\mathbb{R}^{2}$ centered at 0 with radius $a$, and we employ the notation $\Omega_{0}=B(0, a) \backslash \mathcal{O}$. In addition, we assume that the computational domain $\Omega$ is convex and contains $B(0, b)$. We also introduce the notation $\Gamma=\{|\mathbf{x}|=a\}=\partial B(0, a)$. The geometric setting is displayed in Figure 1. The relevant definitions and properties of the involved functions are listed in Appendix A. 


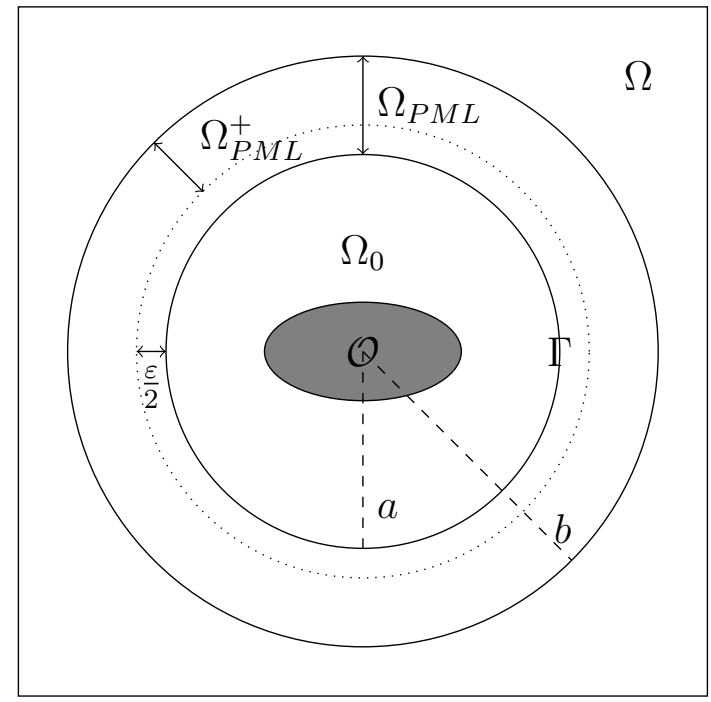

FigURE 1. Illustration of the geometric setting.

As usual we denote by $(\rho, \theta)$ the polar coordinates centred at 0 . According to $[9, \S 3]$ and using the notations from Appendix A, for an arbitrary real number $k$, we consider the boundary value problem

$$
\begin{aligned}
k^{2} d \tilde{d} u+\frac{1}{\rho} \frac{\partial}{\partial \rho}\left(q \rho \frac{\partial u}{\partial \rho}\right)+\frac{1}{q \rho^{2}} \frac{\partial^{2} u}{\partial \theta^{2}} & =d \tilde{d} f \text { in } \Omega \\
u & =0 \text { on } \partial \Omega
\end{aligned}
$$

where the datum $f$ is supposed to be in $L^{2}(\Omega)$. As $d=\tilde{d}=1$ in $\Omega_{0}$, the problem reduces to the Helmholtz equation in $\Omega_{0}$, the PML being situated in $\Omega \backslash \Omega_{0}$. Multiplying the partial differential equation by $q$, we obtain the equivalent problem

$$
\begin{aligned}
k^{2} \tilde{d}^{2} u+\frac{q}{\rho} \frac{\partial}{\partial \rho}\left(q \rho \frac{\partial u}{\partial \rho}\right)+\frac{1}{\rho^{2}} \frac{\partial^{2} u}{\partial \theta^{2}} & =\tilde{d}^{2} f \text { in } \Omega \\
u & =0 \text { on } \partial \Omega .
\end{aligned}
$$

The variational formulation of this problem is obtained by multiplying the partial differential equation by a test-function $\bar{v} \in H_{0}^{1}(\Omega)$ and by using formal integration by parts. Hence we look for $u \in H_{0}^{1}(\Omega)$ solution of

$(2.5)-\int_{\Omega}\left\{q \frac{\partial u}{\partial \rho} \frac{\partial}{\partial \rho}(q \bar{v})+\frac{1}{\rho^{2}} \frac{\partial u}{\partial \theta} \frac{\partial \bar{v}}{\partial \theta}\right\} d x+k^{2} \int_{\Omega} \tilde{d}^{2} u \bar{v} d x=\int_{\Omega} \tilde{d}^{2} f \bar{v} d x, \forall v \in H_{0}^{1}(\Omega)$.

By Leibniz's rule, this formulation is equivalent to

$$
b_{k}(u, v)=-\int_{\Omega} \tilde{d}^{2} f \bar{v} d x, \forall v \in H_{0}^{1}(\Omega),
$$


where the sesquilinear form $b$ is defined by

$$
b_{k}(u, v)=\int_{\Omega}\left\{q^{2} \frac{\partial u}{\partial \rho} \frac{\partial \bar{v}}{\partial \rho}+\frac{1}{\rho^{2}} \frac{\partial u}{\partial \theta} \frac{\partial \bar{v}}{\partial \theta}+q \frac{\partial q}{\partial \rho} \frac{\partial u}{\partial \rho} \bar{v}-k^{2} \tilde{d}^{2} u \bar{v}\right\} d x, \forall u, v \in H_{0}^{1}(\Omega) .
$$

By Theorem 2 of [9], this problem has a unique solution for all real numbers $k$ except possibly a discrete set. For this exceptional discrete set, as we are in a Fredholm setting, uniqueness of a solution is equivalent to existence and uniqueness.

\section{The stability estimate}

Let us start with the following definition.

Definition 3.1. We will say that system (2.6) satisfies the $k$-stability property if there exists $k_{0}>0$ large enough such that for all $k \geq k_{0}$ and all $f \in L^{2}(\Omega)$ any solution $u \in H_{0}^{1}(\Omega)$ of (2.6) satisfies

$$
k\|u\|_{\Omega}+|u|_{1, \Omega} \lesssim\|f\|_{\Omega},
$$

for all $k \geq k_{0}$.

According to this definition, the $k$-stability property directly implies that for $k \geq k_{0}$, problem (2.6) is well-posed since the only solution $u$ of problem (2.6) with $f=0$ is zero.

Let us further remark that once we assume that the $k$-stability property holds, then the best constant in the right-hand side of (3.1) is equivalent to 1. More precisely, we can prove the next result.

Lemma 3.2. Assume that (3.1) holds for all $k \geq k_{0}>0$ and introduce

$$
C_{\mathrm{opt}}(k):=\sup _{f \in L^{2}(\Omega): f \neq 0} \frac{k\left\|u_{f}\right\|_{\Omega}+\left|u_{f}\right|_{1, \Omega}}{\|f\|_{\Omega}},
$$

where $u_{f} \in H_{0}^{1}(\Omega)$ is the unique solution of (2.6). Then one has

$$
C_{\text {opt }}(k) \sim 1, \forall k \geq k_{0} .
$$

Proof. The bound $C_{\text {opt }}(k) \lesssim 1$ being trivial since (3.1) is assumed, we only concentrate on the converse estimate. For that purpose, fix a non zero real valued function $\chi \in \mathcal{D}(\Omega)$ that vanishes in the PML region $\Omega_{P M L}$. Then for all $k \geq k_{0}$, define

$$
u(\mathbf{x})=e^{i k x_{1}} \chi(\mathbf{x}), \forall \mathbf{x} \in \Omega,
$$

where $x_{1}$ is the first component of $\mathbf{x}$, that is considered as solution of (2.6) with $f=$ $\Delta u+k^{2} u$ (as $u$ is zero in the PML). Then direct calculations yield

$$
\|f\|_{\Omega} \sim\|\Delta \chi\|_{\Omega}+k\left\|\partial_{1} \chi\right\|_{\Omega},
$$

and

$$
k\|u\|_{\Omega}+|u|_{1, \Omega} \sim k\|\chi\|_{\Omega}+|\chi|_{1, \Omega} .
$$

Consequently as $\|\chi\|_{\Omega}>0$, we find

$$
\frac{k\|u\|_{\Omega}+|u|_{1, \Omega}}{\|f\|_{\Omega}} \gtrsim 1,
$$


which proves that $C_{\text {opt }}(k) \gtrsim 1$, for all $k \geq k_{0}$.

Let us also notice that any solution $u \in H_{0}^{1}(\Omega)$ of (2.6) satisfies

$$
k^{2} \tilde{d}^{2} u+\frac{1}{\rho} \frac{\partial}{\partial \rho}\left(q^{2} \rho \frac{\partial u}{\partial \rho}\right)+\frac{1}{\rho^{2}} \frac{\partial^{2} u}{\partial \theta^{2}}=\tilde{d}^{2} f+q \frac{\partial q}{\partial \rho} \frac{\partial u}{\partial \rho} \text { in } \mathcal{D}^{\prime}(\Omega),
$$

which is equivalent to (2.3) in the distributional sense. As $q$ tends to 1 as $k$ goes to infinity (cf. Lemma A.2), we deduce that the system

$$
\frac{1}{\rho} \frac{\partial}{\partial \rho}\left(q^{2} \rho \frac{\partial u}{\partial \rho}\right)+\frac{1}{\rho^{2}} \frac{\partial^{2} u}{\partial \theta^{2}}
$$

is strongly elliptic (uniformly in $k$ ) for $k$ large enough. By elliptic regularity, we deduce that, for $k$ large enough, any solution $u \in H_{0}^{1}(\Omega)$ of $(2.6)$ belongs to $H^{2}(\Omega)$ with the estimate

$$
\|u\|_{2, \Omega} \lesssim\|f\|_{\Omega}+k^{2}\|u\|_{\Omega}
$$

Combined with (3.1), we obviously deduce that

$$
\|u\|_{2, \Omega} \lesssim k\|f\|_{\Omega},
$$

for $k$ large enough. Note finally that in such a case (3.3) holds strongly, i.e., as an equality in $L^{2}(\Omega)$.

The goal of this section is to prove the $k$-stability property. This will be made in different steps.

Lemma 3.3. For $k$ large enough, we have

$$
\int_{\Omega_{P M L}^{+}}\left|\frac{\partial u}{\partial \rho}\right|^{2} d x+\int_{\Omega_{P M L}} \tilde{\sigma} k^{2}|u|^{2} d x \lesssim k\|f\|_{\Omega}\|u\|_{\Omega}+\left\|\frac{\partial u}{\partial \rho}\right\|_{\Omega_{P M L}}\|u\|_{\Omega_{P M L}} .
$$

Proof. In (2.6), we take $v=u$ and the imaginary part to obtain

$$
\int_{\Omega}\left\{-\operatorname{Im} q^{2}\left|\frac{\partial u}{\partial \rho}\right|^{2}+k^{2} \operatorname{Im} \tilde{d}^{2}|u|^{2}\right\} d x=\operatorname{Im} \int_{\Omega} \tilde{d}^{2} f \bar{u} d x+\operatorname{Im} \int_{\Omega} q \frac{\partial q}{\partial \rho} \frac{\partial u}{\partial \rho} \bar{u} d x .
$$

By Cauchy-Schwarz's inequality, the fact that $q=\tilde{d}=1$ in $\Omega_{0}$ and Lemma A.3, we find

$$
\int_{\Omega_{P M L}}\left\{-\operatorname{Im} q^{2}\left|\frac{\partial u}{\partial \rho}\right|^{2}+k^{2} \operatorname{Im} \tilde{d}^{2}|u|^{2}\right\} d x \lesssim\|f\|_{\Omega}\|u\|_{\Omega}+\frac{1}{k}\left\|\frac{\partial u}{\partial \rho}\right\|_{\Omega_{P M L}}\|u\|_{\Omega_{P M L}} .
$$

By the identities (A.10) to (A.12), the previous estimate can be equivalently written

$$
\int_{\Omega_{P M L}}\left\{\frac{2 \gamma k \rho \tilde{\sigma}^{\prime}(\rho)}{k^{2}+\sigma^{2}(\rho)}\left|\frac{\partial u}{\partial \rho}\right|^{2}+2 k \tilde{\sigma}|u|^{2}\right\} d x \lesssim\|f\|_{\Omega}\|u\|_{\Omega}+\frac{1}{k}\left\|\frac{\partial u}{\partial \rho}\right\|_{\Omega_{P M L}}\|u\|_{\Omega_{P M L}} .
$$


Since $\tilde{\sigma}^{\prime}$ and $\sigma$ are positive in $\Omega_{P M L}$, in the left-hand side of this estimate, we can reduce the integral over the first summand to $\Omega_{P M L}^{+}$, namely

$$
\int_{\Omega_{P M L}^{+}} \frac{2 \gamma k \rho \tilde{\sigma}^{\prime}(\rho)}{k^{2}+\sigma^{2}(\rho)}\left|\frac{\partial u}{\partial \rho}\right|^{2} d x+\int_{\Omega_{P M L}} 2 k \tilde{\sigma}|u|^{2} d x \lesssim\|f\|_{\Omega}\|u\|_{\Omega}+\frac{1}{k}\left\|\frac{\partial u}{\partial \rho}\right\|_{\Omega_{P M L}}\|u\|_{\Omega_{P M L}} .
$$

By (A.6) and the fact that $\gamma$ tends to 1 as $k$ tends to infinity, we conclude that (3.6) holds for $k$ large enough.

Lemma 3.4. For $k$ large enough, we have

$$
\int_{\Omega}|\nabla u|^{2} d x \lesssim k^{2}\|u\|_{\Omega}^{2}+\|f\|_{\Omega}\|u\|_{\Omega} \lesssim k^{2}\|u\|_{\Omega}^{2}+\|f\|_{\Omega}^{2}
$$

Proof. In (2.6), we take $v=u$ and the real part to obtain

$$
\begin{aligned}
\int_{\Omega}\left\{\operatorname{Re} q^{2}\left|\frac{\partial u}{\partial \rho}\right|^{2}\right. & \left.+\frac{1}{\rho^{2}}\left|\frac{\partial u}{\partial \theta}\right|^{2}\right\} d x \\
& =k^{2} \int_{\Omega} \operatorname{Re} \tilde{d}^{2}|u|^{2} d x-\operatorname{Re} \int_{\Omega} \tilde{d}^{2} f \bar{u} d x-\operatorname{Re} \int_{\Omega} q \frac{\partial q}{\partial \rho} \frac{\partial u}{\partial \rho} \bar{u} d x
\end{aligned}
$$

By Cauchy-Schwarz's inequality, the boundedness of $\tilde{d}$ and $q$ for $k$ large (see Lemma A.2) and Lemma A.3, we obtain

$$
\int_{\Omega}\left\{\operatorname{Re} q^{2}\left|\frac{\partial u}{\partial \rho}\right|^{2}+\frac{1}{\rho^{2}}\left|\frac{\partial u}{\partial \theta}\right|^{2}\right\} d x \lesssim k^{2}\|u\|_{\Omega}^{2}+\|f\|_{\Omega}\|u\|_{\Omega}+\frac{1}{k}\left\|\frac{\partial u}{\partial \rho}\right\|_{\Omega_{P M L}}\|u\|_{\Omega_{P M L}} .
$$

As $q$ tends to 1 as $k$ tends to infinity (see Lemma A.2), for $k$ large enough, we get

$$
\int_{\Omega}\left\{\left|\frac{\partial u}{\partial \rho}\right|^{2}+\frac{1}{\rho^{2}}\left|\frac{\partial u}{\partial \theta}\right|^{2}\right\} d x \lesssim k^{2}\|u\|_{\Omega}^{2}+\|f\|_{\Omega}\|u\|_{\Omega}+\frac{1}{k}\left\|\frac{\partial u}{\partial \rho}\right\|_{\Omega_{P M L}}\|u\|_{\Omega_{P M L}} .
$$

By Young's inequality, we can absorb the last term of this right-hand side, namely

$$
\int_{\Omega}\left\{\left|\frac{\partial u}{\partial \rho}\right|^{2}+\frac{1}{\rho^{2}}\left|\frac{\partial u}{\partial \theta}\right|^{2}\right\} d x \leq C k^{2}\|u\|_{\Omega}^{2}+C\|f\|_{\Omega}\|u\|_{\Omega}+\frac{1}{2 k^{2}}\left\|\frac{\partial u}{\partial \rho}\right\|_{\Omega}^{2}+C^{2}\|u\|_{\Omega}^{2} .
$$

for some $C>0$ independent of $k$. Consequently we get

$$
\left(1-\frac{1}{2 k^{2}}\right) \int_{\Omega}\left\{\left|\frac{\partial u}{\partial \rho}\right|^{2}+\frac{1}{\rho^{2}}\left|\frac{\partial u}{\partial \theta}\right|^{2}\right\} d x \leq C k^{2}\|u\|_{\Omega}^{2}+C\|f\|_{\Omega}\|u\|_{\Omega}+C^{2}\|u\|_{\Omega}^{2},
$$

which yields (3.8) for $k$ large enough since $|\nabla u|^{2}=\left|\frac{\partial u}{\partial \rho}\right|^{2}+\frac{1}{\rho^{2}}\left|\frac{\partial u}{\partial \theta}\right|^{2}$.

In view of this Lemma, we see that the $k$-stability property will be proved if we can estimate $k\|u\|_{\Omega}$. Since Lemma 3.3 gives an estimate of this quantity in $\Omega_{P M L}^{+}$, it remains 
to estimate it in $\Omega \backslash \Omega_{P M L}^{+}$. This is made via a multiplier method. For the cut-off function $\eta$ fixed in the Appendix A, let us introduce the multiplier

$$
m(x)=x \eta(\rho), \forall x \in \Omega
$$

the functions (depending only on the radial variable $\rho$ )

$$
\begin{array}{r}
\alpha=\eta^{\prime}\left(\bar{q}^{2}-2 \operatorname{Re} q^{2}\right)+2 \eta \bar{q} \frac{\partial \bar{q}}{\partial \rho}, \\
\beta=2 \tilde{d}^{2} \eta+\rho \frac{\partial}{\partial \rho}\left(\tilde{d}^{2} \eta\right),
\end{array}
$$

as well as the expressions

$$
\begin{aligned}
\Sigma & =\int_{\Omega}\left(q^{2}-\bar{q}^{2}\right) \eta(\rho) \frac{\partial u}{\partial \rho} \frac{\partial}{\partial \rho}\left(\rho \frac{\partial \bar{u}}{\partial \rho}\right) d x \\
\Sigma_{1} & =\int_{\Omega}\left(\overline{\tilde{d}}^{2}-\tilde{d}^{2}\right) \eta(\rho) \rho \frac{\partial u}{\partial \rho} \bar{u} d x .
\end{aligned}
$$

With these notations, we can prove the following identity with multiplier:

Lemma 3.5. The next identity holds

$$
\begin{aligned}
\int_{\Omega}\left(-k^{2} \beta|u|^{2}+\rho \eta^{\prime}\left|\frac{1}{\rho} \frac{\partial u}{\partial \theta}\right|^{2}-\alpha \rho\left|\frac{\partial u}{\partial \rho}\right|^{2}\right) d x+\int_{\partial \mathcal{O}}|\nabla u \cdot n|^{2} x \cdot n d \sigma(x) \\
=\Sigma-k^{2} \Sigma_{1}+2 \operatorname{Re} \int_{\Omega}\left(\tilde{d}^{2} f+q \frac{\partial q}{\partial \rho} \frac{\partial u}{\partial \rho}\right) \eta \rho \frac{\partial \bar{u}}{\partial \rho} d x
\end{aligned}
$$

Proof. For shortness, let us set $f_{1}=\tilde{d}^{2} f+q \frac{\partial q}{\partial \rho} \frac{\partial u}{\partial \rho}$, then as already said before $u$ satisfies (3.3) or equivalently

$$
k^{2} \tilde{d}^{2} u+\frac{1}{\rho} \frac{\partial}{\partial \rho}\left(q^{2} \rho \frac{\partial u}{\partial \rho}\right)+\frac{1}{\rho^{2}} \frac{\partial^{2} u}{\partial \theta^{2}}=f_{1}
$$

Multiplying this identity by $m \cdot \nabla \bar{u}=\eta \rho \frac{\partial \bar{u}}{\partial \rho}$ and integrating in $\Omega\left(\right.$ meaningful as $u \in H^{2}(\Omega)$ ), we find

$$
k^{2} I_{a}+k^{2} I+J_{a}+J_{\text {rad }}+J_{\text {ang }}=\int_{\Omega} f_{1} \eta \rho \frac{\partial \bar{u}}{\partial \rho} d x
$$


where we have set

$$
\begin{aligned}
J_{\text {rad }} & =\int_{\Omega \backslash \Omega_{0}} \frac{\partial}{\partial \rho}\left(q^{2} \rho \frac{\partial u}{\partial \rho}\right) \eta \frac{\partial \bar{u}}{\partial \rho} d x \\
J_{\text {ang }} & =\int_{\Omega \backslash \Omega_{0}} \frac{1}{\rho} \frac{\partial^{2} u}{\partial \theta^{2}} \eta \frac{\partial \bar{u}}{\partial \rho} d x \\
I & =\int_{\Omega \backslash \Omega_{0}} \tilde{d}^{2} u \eta \rho \frac{\partial \bar{u}}{\partial \rho} d x \\
I_{a} & =\int_{\Omega_{0}} u(m \cdot \nabla \bar{u}) d x \\
J_{a} & =\int_{\Omega_{0}} \Delta u(m \cdot \nabla \bar{u}) d x .
\end{aligned}
$$

We now transform these expressions by using some integrations by parts.

a) Transformation of $I$ : As $\eta$ is zero outside $B(0, b)$, we have

$$
I=\int_{0}^{2 \pi} \int_{a}^{b} \tilde{d}^{2} u \eta \rho^{2} \frac{\partial \bar{u}}{\partial \rho} d \rho d \theta
$$

By integration by parts in $\rho$, we have

$$
I=-\int_{0}^{2 \pi} \int_{a}^{b} \frac{\partial}{\partial \rho}\left(\tilde{d}^{2} u \eta \rho^{2}\right) \bar{u} d \rho d \theta-\int_{\Gamma} a|u|^{2} d \sigma(x),
$$

the boundary term being zero since $\eta(b)=0$. By Leibniz's rule, we deduce that

$$
\begin{aligned}
I & =-\int_{0}^{2 \pi} \int_{a}^{b} \frac{\partial}{\partial \rho}\left(\tilde{d}^{2} \eta \rho^{2}\right)|u|^{2} d \rho d \theta \\
& -\int_{0}^{2 \pi} \int_{a}^{b} \tilde{d}^{2} \eta \rho^{2} \frac{\partial u}{\partial \rho} \bar{u} d \rho d \theta-\int_{\Gamma} a|u|^{2} d \sigma(x) .
\end{aligned}
$$

The second term of this right-hand side would be equal to $-\bar{I}$ if $\tilde{d}^{2}$ would be real, hence by introducing $\Sigma_{1}$, we find that

$$
2 \operatorname{Re} I=-\int_{\Omega \backslash \Omega_{0}} \beta|u|^{2} d x+\Sigma_{1}-\int_{\Gamma} a|u|^{2} d \sigma(x) .
$$

b) Transformation of $I_{a}$ : By the Green formula, we have

$$
\begin{aligned}
2 \operatorname{Re} I_{a} & =2 \operatorname{Re} \int_{\Omega_{0}} u(m \cdot \nabla \bar{u}) d x \\
& =\int_{\Omega_{0}} m \cdot \nabla|u|^{2} d x \\
& =-\int_{\Omega_{0}} 2|u|^{2} d x+\int_{\partial \Omega_{0}} m \cdot n|u|^{2} d \sigma(x) .
\end{aligned}
$$


Since $u=0$ on $\partial \mathcal{O}$, we have

$$
2 \operatorname{Re} I_{a}=-\int_{\Omega_{0}} \beta|u|^{2} d x+\int_{\Gamma} a|u|^{2} d \sigma(x) .
$$

c) Transformation of $J_{\text {ang }}$ : As before we have

$$
J_{\text {ang }}=\int_{0}^{2 \pi} \int_{a}^{b} \frac{\partial^{2} u}{\partial \theta^{2}} \eta \frac{\partial \bar{u}}{\partial \rho} d \rho d \theta
$$

and by integration by parts in $\theta$, we find

$$
J_{\text {ang }}=-\int_{0}^{2 \pi} \int_{a}^{b} \frac{\partial u}{\partial \theta} \eta \frac{\partial^{2} \bar{u}}{\partial \theta \partial \rho} d \rho d \theta
$$

Since

we then have

$$
\frac{\partial}{\partial \rho}\left|\frac{\partial u}{\partial \theta}\right|^{2}=2 \operatorname{Re}\left(\frac{\partial u}{\partial \theta} \frac{\partial^{2} \bar{u}}{\partial \theta \partial \rho}\right)
$$

$$
2 \operatorname{Re} J_{a n g}=-\int_{0}^{2 \pi} \int_{a}^{b} \eta \frac{\partial}{\partial \rho}\left|\frac{\partial u}{\partial \theta}\right|^{2} d \rho d \theta .
$$

By integration by parts in $\rho$, we deduce that

$$
2 \operatorname{Re} J_{\text {ang }}=\int_{\Omega_{0}} \rho \eta^{\prime}\left|\frac{1}{\rho} \frac{\partial u}{\partial \theta}\right|^{2} d x+\int_{\Gamma} a\left|\frac{1}{\rho} \frac{\partial u}{\partial \theta}\right|^{2} d \sigma(x) .
$$

d) Transformation of $J_{\text {rad }}$ : As before we have

$$
J_{\text {rad }}=\int_{0}^{2 \pi} \int_{a}^{b} \frac{\partial}{\partial \rho}\left(q^{2} \rho \frac{\partial u}{\partial \rho}\right) \eta \rho \frac{\partial \bar{u}}{\partial \rho} d \rho d \theta
$$

and an integration by parts in $\rho$ yields

$$
\begin{aligned}
J_{\text {rad }} & =-\int_{0}^{2 \pi} \int_{a}^{b} q^{2} \rho \frac{\partial u}{\partial \rho} \frac{\partial}{\partial \rho}\left(\eta \rho \frac{\partial \bar{u}}{\partial \rho}\right) d \rho d \theta-\int_{\Gamma} a\left|\frac{\partial u}{\partial \rho}\right|^{2} d \sigma(x) \\
& =-\int_{0}^{2 \pi} \int_{a}^{b} q^{2} \eta^{\prime}\left|\rho \frac{\partial u}{\partial \rho}\right|^{2} d \rho d \theta \\
& -\int_{0}^{2 \pi} \int_{a}^{b} q^{2} \eta \rho \frac{\partial u}{\partial \rho} \frac{\partial}{\partial \rho}\left(\rho \frac{\partial \bar{u}}{\partial \rho}\right) d \rho d \theta-\int_{\Gamma} a\left|\frac{\partial u}{\partial \rho}\right|^{2} d \sigma(x) .
\end{aligned}
$$

This can be equivalently written as

$$
J_{\text {rad }}=-K-\int_{0}^{2 \pi} \int_{0}^{b} q^{2} \eta^{\prime}\left|\rho \frac{\partial u}{\partial \rho}\right|^{2} d \rho d \theta-\int_{\Gamma} a\left|\frac{\partial u}{\partial \rho}\right|^{2} d \sigma(x)
$$

where we have set

$$
K:=\int_{0}^{2 \pi} \int_{0}^{b} q^{2} \eta w \frac{\partial \bar{w}}{\partial \rho} d \rho d \theta \quad \text { and } \quad w:=\rho \frac{\partial u}{\partial \rho} .
$$


Introducing $\Sigma$, we see that

$$
K=\Sigma+\int_{0}^{2 \pi} \int_{0}^{b} \bar{q}^{2} \eta w \frac{\partial \bar{w}}{\partial \rho} d \rho d \theta
$$

hence integrating by parts in $\rho$ in the second term of this right-hand side, we get

$$
\begin{aligned}
K & =\Sigma-\int_{0}^{2 \pi} \int_{0}^{b} \frac{\partial}{\partial \rho}\left(\bar{q}^{2} \eta w\right) \bar{w} d \rho d \theta-\int_{\Gamma} a\left|\frac{\partial u}{\partial \rho}\right|^{2} d \sigma(x) \\
& =\Sigma-\bar{K}-\int_{0}^{2 \pi} \int_{0}^{b} \frac{\partial}{\partial \rho}\left(\bar{q}^{2} \eta\right)|w|^{2} d \rho d \theta-\int_{\Gamma} a\left|\frac{\partial u}{\partial \rho}\right|^{2} d \sigma(x) .
\end{aligned}
$$

This yields

$$
2 \operatorname{Re} K=\Sigma-\int_{0}^{2 \pi} \int_{0}^{b} \frac{\partial}{\partial \rho}\left(\bar{q}^{2} \eta\right)|w|^{2} d \rho d \theta-\int_{\Gamma} a\left|\frac{\partial u}{\partial \rho}\right|^{2} d \sigma(x) .
$$

Taking the real part of the identity (3.18), we conclude that

$$
2 \operatorname{Re} J_{\text {rad }}=-\Sigma-\int_{\Omega} \alpha \rho\left|\frac{\partial u}{\partial \rho}\right|^{2} d x-\int_{\Gamma} a\left|\frac{\partial u}{\partial \rho}\right|^{2} d \sigma(x) .
$$

e) Transformation of $J_{a}$ : By integration by parts, we have

$$
\begin{aligned}
J_{a} & =\int_{\Omega_{0}} \Delta u(m \cdot \nabla \bar{u}) d x \\
& =-\int_{\Omega_{0}} \nabla u \cdot \nabla(m \cdot \nabla \bar{u}) d x+\int_{\Gamma} \nabla u \cdot n(m \cdot \nabla \bar{u}) d \sigma(x)+\int_{\partial \mathcal{O}} \nabla u \cdot n(m \cdot \nabla \bar{u}) d \sigma(x),
\end{aligned}
$$

We recall that $m=x$ in $\Omega_{0}$. In addition, since $u=0$ on $\partial \mathcal{O}$, we also have $\nabla u \cdot t=0$ on $\partial \mathcal{O}$ for the unit tangent vector $t$. It follows that

$$
m \cdot \nabla \bar{u}=m \cdot n \nabla \bar{u} \cdot n+m \cdot t \nabla \bar{u} \cdot t=\nabla \bar{u} \cdot n x \cdot n,
$$

and

$$
\nabla u \cdot n(m \cdot \nabla \bar{u})=|\nabla u \cdot n|^{2} x \cdot n,
$$

on $\partial \mathcal{O}$. On the other hand, Rellich's identity yields that

$$
2 \operatorname{Re} \int_{\Omega_{0}} \nabla u \cdot \nabla(m \cdot \nabla \bar{u})=\int_{\partial \Omega_{0}}|\nabla u|^{2} x \cdot n=\int_{\Gamma}|\nabla u|^{2} x \cdot n+\int_{\partial \mathcal{O}}|\nabla u \cdot n|^{2} x \cdot n .
$$

Recalling that $m=x$ in $\Omega_{0}$ and $\nabla u \cdot t=0$ on $\partial \mathcal{O}$, and using Rellich's identity, we find that

$$
\begin{aligned}
2 \operatorname{Re} J_{a} & =\int_{\partial \mathcal{O}}|\nabla u \cdot n|^{2} x \cdot n-\int_{\Gamma}|\nabla u|^{2} x \cdot n+2 \int_{\Gamma} \nabla u \cdot n(m \cdot \nabla \bar{u}) \\
& =\int_{\partial \mathcal{O}}|\nabla u \cdot n|^{2} x \cdot n+\int_{\Gamma} a\left|\frac{\partial u}{\partial \rho}\right|^{2}-\int_{\Gamma} a\left|\frac{1}{\rho} \frac{\partial u}{\partial \theta}\right|^{2} .
\end{aligned}
$$

Coming back to (3.14), taking the real part and using (3.15), (3.16), (3.17), (3.19) and (3.20), we arrive at (3.13).

The previous Lemmas allow to conclude the 
Theorem 3.6. System (2.6) satisfies the k-stability property.

Proof. We first look at the behavior of $\beta$ as $k$ is large. By Leibniz's rule, we have

$$
\beta=\beta_{0}+\rho \tilde{d}^{2} \eta^{\prime}
$$

with

$$
\beta_{0}=2\left(\tilde{d}^{2}+\rho \tilde{d} \frac{\partial \tilde{d}}{\partial \rho}\right) \eta
$$

With this splitting, (3.13) implies that

$$
\begin{aligned}
& \int_{\Omega}\left(k^{2} \beta_{0}|u|^{2}-\rho \eta^{\prime}\left|\frac{1}{\rho} \frac{\partial u}{\partial \theta}\right|^{2}\right) d x \\
& \quad \leq-\Sigma+k^{2} \Sigma_{1}-2 \operatorname{Re} \int_{\Omega} f_{1} \eta \rho \frac{\partial \bar{u}}{\partial \rho} d x-\int_{\Omega} \alpha \rho\left|\frac{\partial u}{\partial \rho}\right|^{2} d x-k^{2} \int_{\Omega_{P M L}^{+}} \rho \tilde{d}^{2} \eta^{\prime}|u|^{2} d x .
\end{aligned}
$$

Since $\tilde{d}^{2}$ tends to 1 as $k$ goes to infinity and $\frac{\partial \tilde{d}}{\partial \rho}=\frac{i \tilde{\sigma}^{\prime}}{k}$ tends to 0 as $k$ goes to infinity, we directly see that

$$
\operatorname{Re} \beta_{0} \geq \eta \text {, for } k \text { large enough. }
$$

Using this property, the boundedness of $\tilde{d}$ and the fact that $\eta^{\prime} \leq 0$ in (3.21), we find that

$$
\begin{array}{rl}
k^{2} \int_{\Omega} \eta|u|^{2} & d x \\
+\int_{\Omega}|\alpha| \rho\left|\frac{\partial u}{\partial \rho}\right|^{2} d x+k^{2} \int_{\Omega_{P M L}^{+}}|u|^{2} d x
\end{array}
$$

for $k$ large enough. Now by the definition of $\alpha$ and Lemmas A.2 and A.3, we have

$$
\begin{aligned}
\int_{\Omega}|\alpha| \rho\left|\frac{\partial u}{\partial \rho}\right|^{2} d x+k^{2} \int_{\Omega_{P M L}^{+}}|u|^{2} d x \lesssim \int_{\Omega_{P M L}^{+}} & \left(\left|\frac{\partial u}{\partial \rho}\right|^{2}+k^{2}|u|^{2}\right) d x \\
& +\frac{1}{k} \int_{\Omega_{P M L}}\left|\frac{\partial u}{\partial \rho}\right|^{2} d x .
\end{aligned}
$$

With the help of (3.6), we then obtain

$$
\begin{aligned}
\int_{\Omega}|\alpha| \rho\left|\frac{\partial u}{\partial \rho}\right|^{2} d x+k^{2} \int_{\Omega_{P M L}^{+}}|u|^{2} d x \\
\quad \lesssim k\|f\|_{\Omega}\|u\|_{\Omega}+\left\|\frac{\partial u}{\partial \rho}\right\|_{\Omega_{P M L}}\|u\|_{\Omega_{P M L}}+\frac{1}{k}\left\|\frac{\partial u}{\partial \rho}\right\|_{\Omega_{P M L}}^{2}
\end{aligned}
$$


This estimate in (3.23) leads to

$$
\begin{array}{rl}
k^{2} \int_{\Omega} \eta|u|^{2} & d x \lesssim|\Sigma|+k^{2}\left|\Sigma_{1}\right|+\left\|f_{1}\right\|_{\Omega}\|\nabla u\|_{\Omega} \\
& +k\|f\|_{\Omega}\|u\|_{\Omega}+\left\|\frac{\partial u}{\partial \rho}\right\|_{\Omega_{P M L}}\|u\|_{\Omega_{P M L}}+\frac{1}{k}\left\|\frac{\partial u}{\partial \rho}\right\|_{\Omega_{P M L}}^{2},
\end{array}
$$

for $k$ large enough.

It then remains to estimate $|\Sigma|$ and $k^{2}\left|\Sigma_{1}\right|$.

i) By the definition (3.12) and (A.16), it holds

$$
k^{2}\left|\Sigma_{1}\right| \leq 4 \int_{\Omega_{P M L}} \tilde{\sigma}^{1 / 2}\left|\frac{\partial u}{\partial \rho}\right| k \tilde{\sigma}^{1 / 2}|u| d x .
$$

Cauchy-Schwarz's inequality and the boundedness of $\tilde{\sigma}^{1 / 2}$ then lead to

$$
k^{2}\left|\Sigma_{1}\right| \lesssim\left\|\frac{\partial u}{\partial \rho}\right\|_{\Omega_{P M L}}\left\|k \tilde{\sigma}^{1 / 2} u\right\|_{\Omega_{P M L}}
$$

Using Young's inequality (with an arbitrary $\lambda>0$ ) and (3.6) we infer

$$
\begin{aligned}
k^{2}\left|\Sigma_{1}\right| & \lesssim \lambda\left\|\frac{\partial u}{\partial \rho}\right\|_{\Omega_{P M L}}^{2}+\frac{1}{\lambda} \int_{\Omega_{P M L}} \tilde{\sigma} k^{2}|u|^{2} d x \\
& \lesssim \lambda\left\|\frac{\partial u}{\partial \rho}\right\|_{\Omega_{P M L}}^{2}+\frac{1}{\lambda}\left(k\|f\|_{\Omega}\|u\|_{\Omega}+\left\|\frac{\partial u}{\partial \rho}\right\|_{\Omega_{P M L}}\|u\|_{\Omega_{P M L}}\right) .
\end{aligned}
$$

For the second term of this right-hand side using Young's inequality we find

$$
\begin{aligned}
|\Sigma| & \lesssim \frac{1}{k}\left\|\frac{\partial u}{\partial \rho}\right\|_{\Omega_{P M L}}^{2} \\
& +\frac{1}{\delta k \sqrt{k}} \int_{\Omega_{P M L}} \tilde{\sigma}^{\prime}\left|\frac{\partial u}{\partial \rho}\right|^{2} d x \\
& +\frac{\delta}{\sqrt{k}} \int_{\Omega_{P M L}}\left|\frac{\partial^{2} u}{\partial \rho^{2}}\right|^{2} d x
\end{aligned}
$$

for all $\delta>0$. Using (3.7), the fact that $\gamma$ tends to 1 as $k$ goes to infinity and the property $\frac{k}{k^{2}+\sigma^{2}} \geq \frac{1}{k}$ valid for $k$ large enough, we find

$$
\begin{aligned}
|\Sigma| & \lesssim \frac{1}{k}\left\|\frac{\partial u}{\partial \rho}\right\|_{\Omega_{P M L}}^{2} \\
& +\frac{1}{\delta \sqrt{k}}\left(\|f\|_{\Omega}\|u\|_{\Omega}+\frac{1}{k}\left\|\frac{\partial u}{\partial \rho}\right\|_{\Omega_{P M L}}\|u\|_{\Omega_{P M L}}\right) \\
& +\frac{\delta}{\sqrt{k}} \int_{\Omega_{P M L}}\left|\frac{\partial^{2} u}{\partial \rho^{2}}\right|^{2} d x
\end{aligned}
$$


for all $\delta>0$ and for $k$ large enough. For the last term of this right-hand side, using the estimate (3.4), we arrive at

$$
\begin{aligned}
|\Sigma| & \lesssim \frac{1}{k}\left\|\frac{\partial u}{\partial \rho}\right\|_{\Omega_{P M L}}^{2} \\
& +\frac{1}{\delta \sqrt{k}}\left(\|f\|_{\Omega}\|u\|_{\Omega}+\frac{1}{k}\left\|\frac{\partial u}{\partial \rho}\right\|_{\Omega_{P M L}}\|u\|_{\Omega_{P M L}}\right) \\
& +\frac{\delta}{\sqrt{k}}\left(\|f\|_{\Omega}^{2}+k^{4}\|u\|_{\Omega}^{2}\right) .
\end{aligned}
$$

This estimate and (3.25) in (3.24)

$$
\begin{aligned}
k^{2} \int_{\Omega} \eta|u|^{2} d x & \lesssim\left\|f_{1}\right\|_{\Omega}\|\nabla u\|_{\Omega} \\
& +k\|f\|_{\Omega}\|u\|_{\Omega}+\left\|\frac{\partial u}{\partial \rho}\right\|_{\Omega_{P M L}}\|u\|_{\Omega_{P M L}}+\left(\lambda+\frac{1}{k}\right)\|\nabla u\|_{\Omega_{P M L}}^{2} \\
& +\left(\frac{k}{\lambda}+\frac{1}{\delta \sqrt{k}}\right)\|f\|_{\Omega}\|u\|_{\Omega}+\left(\frac{1}{\lambda}+\frac{1}{\delta k^{\frac{3}{2}}}\right)\left\|\frac{\partial u}{\partial \rho}\right\|_{\Omega_{P M L}}\|u\|_{\Omega_{P M L}} \\
& +\frac{\delta}{\sqrt{k}}\|f\|_{\Omega}^{2}+\delta k^{\frac{7}{2}}\|u\|_{\Omega}^{2},
\end{aligned}
$$

for $k$ large enough.

Comparing this estimate with (3.6) and recalling that $\eta=1$ in $\Omega \backslash \Omega_{P M L}^{+}$and (A.6) for large $k$, we have shown that

$$
\begin{aligned}
k^{2} \int_{\Omega}|u|^{2} d x & \leq C\left(\left\|f_{1}\right\|_{\Omega}\|\nabla u\|_{\Omega}\right. \\
& +k\|f\|_{\Omega}\|u\|_{\Omega}+\left\|\frac{\partial u}{\partial \rho}\right\|_{\Omega_{P M L}}\|u\|_{\Omega_{P M L}}+\left(\lambda+\frac{1}{k}\right)\|\nabla u\|_{\Omega_{P M L}}^{2} \\
& +\left(\frac{k}{\lambda}+\frac{1}{\delta \sqrt{k}}\right)\|f\|_{\Omega}\|u\|_{\Omega}+\left(\frac{1}{\lambda}+\frac{1}{\delta k^{\frac{3}{2}}}\right)\left\|\frac{\partial u}{\partial \rho}\right\|_{\Omega_{P M L}}\|u\|_{\Omega_{P M L}} \\
& \left.+\frac{\delta}{\sqrt{k}}\|f\|_{\Omega}^{2}+\delta k^{\frac{7}{2}}\|u\|_{\Omega}^{2}\right)
\end{aligned}
$$

for $k$ large enough and some positive constant $C$ independent of $k$. We now chose $\delta>0$ so that

$$
C \delta k^{\frac{7}{2}}=\frac{k^{2}}{2}
$$

or equivalently

$$
\delta=\frac{k^{-\frac{3}{2}}}{2 C}
$$


With this choice we find

$$
\begin{aligned}
k^{2} \int_{\Omega}|u|^{2} d x & \lesssim\left\|f_{1}\right\|_{\Omega}\|\nabla u\|_{\Omega} \\
& +k\left(1+\frac{1}{\lambda}\right)\|f\|_{\Omega}\|u\|_{\Omega}+\left(1+\frac{1}{\lambda}\right)\left\|\frac{\partial u}{\partial \rho}\right\|_{\Omega_{P M L}}\|u\|_{\Omega_{P M L}} \\
& +\left(\lambda+\frac{1}{k}\right)\|\nabla u\|_{\Omega_{P M L}}^{2}+\left\|\frac{\partial u}{\partial \rho}\right\|_{\Omega_{P M L}}\|u\|_{\Omega_{P M L}}+\frac{1}{k^{2}}\|f\|_{\Omega}^{2},
\end{aligned}
$$

for $k$ large enough. Recalling the definition of $f_{1}$ and Lemma A.3, we get

$$
\left\|f_{1}\right\|_{\Omega} \lesssim\|f\|_{\Omega}+\frac{1}{k}\|\nabla u\|_{\Omega}
$$

and consequently

$$
\begin{aligned}
k^{2} \int_{\Omega}|u|^{2} d x & \lesssim\|f\|_{\Omega}\|\nabla u\|_{\Omega}+\left(\lambda+\frac{1}{k}\right)\|\nabla u\|_{\Omega}^{2} \\
& +k\left(1+\frac{1}{\lambda}\right)\|f\|_{\Omega}\|u\|_{\Omega}+\left(1+\frac{1}{\lambda}\right)\|\nabla u\|_{\Omega}\|u\|_{\Omega}+\|f\|_{\Omega}^{2} .
\end{aligned}
$$

for $k$ large enough. By Young's inequality, this estimate implies that

$$
\begin{aligned}
k^{2} \int_{\Omega}|u|^{2} d x & \leq \frac{C}{\mu}\|f\|_{\Omega}^{2}+\mu\|\nabla u\|_{\Omega}^{2}+C\left(\lambda+\frac{1}{k}\right)\|\nabla u\|_{\Omega}^{2} \\
& +\frac{C}{\mu_{1}}\left(1+\frac{1}{\lambda}\right)\|f\|_{\Omega}^{2}+\mu_{1}\left(1+\frac{1}{\lambda}\right) k^{2}\|u\|_{\Omega}^{2} \\
& +\frac{C}{\mu_{2} k^{2}}\left(1+\frac{1}{\lambda}\right)\|\nabla u\|_{\Omega}^{2}+\mu_{2}\left(1+\frac{1}{\lambda}\right) k^{2}\|u\|_{\Omega}^{2}+C\|f\|_{\Omega}^{2},
\end{aligned}
$$

for $k$ large enough, for any positive real numbers $\mu, \mu_{1}$ and $\mu_{2}$ and a positive constant $C$ independent of $k$ (and $\mu, \mu_{1}$ and $\left.\mu_{2}\right)$. Choosing $\mu_{1}=\mu_{2}=(4(1+1 / \lambda))^{-1}$, we find that

$$
k^{2} \int_{\Omega}|u|^{2} d x \leq C\left(1+\frac{1}{\mu}\left(1+\frac{1}{\lambda}\right)^{2}\right)\|f\|_{\Omega}^{2}+\left(\mu+C \lambda+\frac{C}{k}\left(1+\frac{1}{\lambda}\right)^{2}\right)\|\nabla u\|_{\Omega}^{2},
$$

for $k$ large enough, for any positive real numbers $\mu, \lambda$ and a positive constant $C$ independent of $k, \mu, \lambda$. At this stage we take advantage of (3.8) to obtain

$$
\begin{aligned}
k^{2} \int_{\Omega}|u|^{2} d x \leq C\left(\left(1+\frac{1}{\mu}(1\right.\right. & \left.\left.+\frac{1}{\lambda}\right)^{2}+\mu+C \lambda+\frac{C}{k}\left(1+\frac{1}{\lambda}\right)^{2}\right)\|f\|_{\Omega}^{2} \\
& +(\mu+C \lambda) k^{2}\|u\|_{\Omega}^{2}+k C\left(1+\frac{1}{\lambda}\right)^{2}\|u\|_{\Omega}^{2} .
\end{aligned}
$$

Choosing $\mu=\frac{1}{4}$ and $\lambda=\frac{1}{4 C}$, we find that

$$
k^{2} \int_{\Omega}|u|^{2} d x \leq C\|f\|_{\Omega}^{2}+C k\|u\|_{\Omega}^{2}
$$

for $k$ large enough and a positive constant $C$ independent of $k$. As for $k$ large enough $C k \leq \frac{k^{2}}{2}$, we have proved that

$$
k\|u\|_{\Omega} \lesssim\|f\|_{\Omega}
$$


for $k$ large enough. Coming back to (3.8), we conclude that

$$
\int_{\Omega}|\nabla u|^{2} d x \lesssim\|f\|_{\Omega}^{2}
$$

for $k$ large enough.

\section{Comparison With A SPONGe LAYER}

The boundary value problem corresponding to a sponge layer consists in looking at $u_{\text {sponge }}$ solution of

$$
\begin{aligned}
L_{\text {sponge }} u_{\text {sponge }} & =f \text { in } \Omega, \\
u_{\text {sponge }} & =0 \text { on } \partial \Omega,
\end{aligned}
$$

where the operator $L_{\text {sponge }}$ is defined by

$$
L_{\text {sponge }} v=\Delta v+\left(k^{2}+2 i \tilde{\sigma} k\right) v=\frac{1}{\rho} \frac{\partial}{\partial \rho}\left(\rho \frac{\partial v}{\partial \rho}\right)+\frac{1}{\rho^{2}} \frac{\partial^{2} v}{\partial \theta^{2}}+\left(k^{2}+2 i \tilde{\sigma} k\right) v .
$$

This problem (4.1) enters in the framework developed recently in [7] if the boundary of $\Omega$ is $C^{1,1}$ or if it is a convex polygon, since it satisfies the assumption of Section 2 of that paper [7] (with the choices $\mathcal{L}_{0}=-I d, \mathcal{L}_{1}=-2 \tilde{\sigma} I d$, and $\mathcal{L}_{2}=-\Delta$ ), and since its variational formulation is given by

$$
a_{\text {sponge }}\left(u_{\text {sponge }}, v\right)=-\int_{\Omega} f \bar{v} d x, \forall v \in H_{0}^{1}(\Omega),
$$

where the sesquilinear form $a_{\text {sponge }}(\cdot, \cdot)$ is defined by

$$
a_{\text {sponge }}(v, w)=\int_{\Omega}\left(\nabla v \cdot \nabla \bar{w}-\left(k^{2}+2 i \tilde{\sigma} k\right) v \bar{w}\right) d x, \forall v, w \in H_{0}^{1}(\Omega) .
$$

This sesquilinear form trivially satisfies

$$
\left|a_{\text {sponge }}(v, w)\right| \lesssim|||v||||||w|||, \forall v, w \in H_{0}^{1}(\Omega),
$$

where

and

$$
\||v|\|=\left(k^{2}\|v\|_{\Omega}^{2}+|v|_{1, \Omega}^{2}\right)^{\frac{1}{2}}
$$

$$
\operatorname{Re} a_{\text {sponge }}(v, v) \geq|v|_{1, \Omega}^{2}-k^{2}\|v\|_{\Omega}^{2}, \forall v \in H_{0}^{1}(\Omega) .
$$

Consequently the associated operator $A_{\text {sponge }}$ is a Fredholm operator from $H_{0}^{1}(\Omega)$ into $H^{-1}(\Omega)$, therefore it is an isomorphism if and only if it is injective. But the injectivity is not difficult to show because $u \in H_{0}^{1}(\Omega)$ solution of (4.3) with $f=0$ satisfies in particular

$$
a_{\text {sponge }}(u, u)=0 \text {, }
$$

and taking the imaginary part we get

$$
u=0 \text { on } \Omega_{P M L} .
$$

Since $u$ also satisfies

$$
\Delta u+\left(k^{2}+2 i \tilde{\sigma} k\right) u=0 \text { in } \Omega,
$$


by Holmgrem's theorem we deduce that $u=0$.

In order to compare (4.1) with (3.3), we rewrite (3.3) as

$$
L_{\mathrm{PML}} u=\tilde{d}^{2} f
$$

with

$$
L_{\mathrm{PML}} v=k^{2} \tilde{d}^{2} v+\frac{q^{2}}{\rho} \frac{\partial}{\partial \rho}\left(\rho \frac{\partial v}{\partial \rho}\right)+\frac{1}{\rho^{2}} \frac{\partial^{2} v}{\partial \theta^{2}}+q \frac{\partial q}{\partial \rho} \frac{\partial v}{\partial \rho},
$$

We can look at $u$ as solution of

$$
\begin{aligned}
L_{\text {sponge }} u & =f^{(k)} \text { in } \Omega, \\
u & =0 \text { on } \partial \Omega,
\end{aligned}
$$

where $f^{(k)}=L_{\text {sponge }} u-L_{\mathrm{PML}} u+\tilde{d}^{2} f$ and consequently

$$
f^{(k)}=\tilde{d}^{2} f+\left(1-q^{2}\right) \frac{1}{\rho} \frac{\partial}{\partial \rho}\left(\rho \frac{\partial u}{\partial \rho}\right)+\left(\left(k^{2}+2 i \tilde{\sigma} k\right)-k^{2} \tilde{d}^{2}\right) u-q \frac{\partial q}{\partial \rho} \frac{\partial u}{\partial \rho} .
$$

Let us now estimate the $L^{2}$-norm of $f^{(k)}$.

Lemma 4.1. For $k$ large enough, it holds

$$
\left\|f^{(k)}\right\|_{\Omega} \lesssim\|f\|_{\Omega}
$$

Proof. As $\tilde{d}$ is uniformly bounded in $\Omega$, it suffices to estimate the $L^{2}$-norm of the three other terms of the right-hand side of (4.6). For the second term of (4.6), by (A.19), we have

$$
\left\|\left(1-q^{2}\right) \frac{1}{\rho} \frac{\partial}{\partial \rho}\left(\rho \frac{\partial u}{\partial \rho}\right)\right\|_{\Omega} \lesssim \frac{1}{k}\left\|\frac{1}{\rho} \frac{\partial}{\partial \rho}\left(\rho \frac{\partial u}{\partial \rho}\right)\right\|_{\Omega_{P M L}} \lesssim \frac{1}{k}\|u\|_{2, \Omega_{P M L}} .
$$

By (3.5), we conclude that

$$
\left\|\left(1-q^{2}\right) \frac{1}{\rho} \frac{\partial}{\partial \rho}\left(\rho \frac{\partial u}{\partial \rho}\right)\right\|_{\Omega} \lesssim\|f\|_{\Omega}
$$

for $k$ large enough.

The definition of $\tilde{d}$ shows

$$
\left(k^{2}+2 i \tilde{\sigma} k\right)-k^{2} \tilde{d}^{2}=\tilde{\sigma}^{2} .
$$

This identity and the bound (3.1) show that the the third term of (4.6) satisfies

$$
\left\|\left(\left(k^{2}+2 i \tilde{\sigma} k\right)-k^{2} \tilde{d}^{2}\right) u\right\|_{\Omega} \lesssim\|u\|_{\Omega} \lesssim\|f\|_{\Omega},
$$

for $k$ large enough.

For the last term of (4.6), using Lemma A.3 and again (3.1), we directly conclude that

$$
\left\|q \frac{\partial q}{\partial \rho} \frac{\partial u}{\partial \rho}\right\|_{\Omega} \lesssim \frac{1}{k}|u|_{1, \Omega} \lesssim\|f\|_{\Omega},
$$

for $k$ large enough. This estimate, (4.8), and (4.9) lead to the asserted estimate. 
At this stage, we can look at $u \in H_{0}^{1}(\Omega)$ as the unique solution of (4.1) with a datum $f^{(k)}$ instead of $f$. The $L^{2}$ norm of $f^{(k)}$ is uniformly bounded in $k$. Consequently applying Theorem 1 of [7], we directly get the next result.

Theorem 4.2. Let $\gamma$ be a natural number and assume that the boundary of $\Omega$ is of class $C^{\gamma+1,1}$. Then for $k$ large enough, for all $\ell \in\{0, \cdots, \gamma\}$, the unique solution $u \in H_{0}^{1}(\Omega)$ of (2.6) admits the splitting

$$
u=\sum_{j=0}^{\ell-1} k^{j} u_{j}^{(k)}+r_{\ell}^{(k)}
$$

where $u_{j}^{(k)} \in H^{j+2}(\Omega)$ with

$$
\left\|u_{j}^{(k)}\right\|_{j+2, \Omega} \lesssim\|f\|_{\Omega}
$$

for $0 \leq j \leq \ell-1$ and $r_{\ell}^{(k)} \in H^{\ell+2}(\Omega)$ with

$$
\left\|r_{\ell}^{(k)}\right\|_{\ell+2, \Omega} \lesssim k^{\ell+1}\|f\|_{\Omega}
$$

Remark 4.3. This result remains valid for a convex polygon with $\gamma=0$.

\section{Finite element discretizations}

5.1. $h p$-FEM. Here we want to take advantage of the splitting from Theorem 4.2 to derive stability conditions and error estimates for $h p$ finite element discretizations of (2.6).

We look for a finite element approximation $u_{h, p}$ to $u$. To this end, we consider a family of regular (in Ciarlet's sense) meshes $\left\{\mathcal{T}_{h}\right\}_{h}$ of $\Omega$, where each mesh is made of triangular elements $K$. To simplify the analysis, we assume that the boundary of $\Omega$ is exactly triangulated, and therefore, we consider curved Lagrange finite elements [3]. Also, for each element $K$, we denote by $\mathcal{F}_{K}$ the mapping taking the reference element $\hat{K}$ to $K$.

Then, for all $p \leq \gamma+1$, the finite element approximation space $V_{h, p}$ is defined as

$$
V_{h, p}=\left\{v_{h, p} \in H_{0}^{1}(\Omega)\left|v_{h, p}\right|_{K} \circ \mathcal{F}_{K}^{-1} \in \mathbb{P}_{p}(\hat{K}) \forall K \in \mathcal{T}_{h}\right\}
$$

where $\mathbb{P}_{p}(\hat{K})$ stands for the set of polynomials of total degree less than or equal to $p$.

As the family of meshes is regular, for each $v \in H^{l+1}(\Omega) \cap H_{0}^{1}(\Omega)^{S}(0 \leq l \leq p)$, there exists an element $\mathcal{I}_{h, p} v \in V_{h, p}$ such that

$$
\left|v-\mathcal{I}_{h, p} v\right|_{j, \Omega} \lesssim h^{l+1-j}\|v\|_{l+1, \Omega}, \quad(0 \leq j \leq l) .
$$

We refer the reader to [3, Corollary 5.2] (see also [8]).

Then a finite element approximation of $u$ is obtained by looking for $u_{h, p} \in V_{h, p}$ such that

$$
b_{k}\left(u_{h, p}, v_{h, p}\right)=-\int_{\Omega} \tilde{d}^{2} f \bar{v}_{h, p} d x, \quad \forall v_{h, p} \in V_{h, p} .
$$


5.1.1. Asymptotic error estimate. Now we are ready to prove a convergence result in an appropriate asymptotic range.

Theorem 5.1. Assume that the boundary of $\Omega$ is of class $C^{\gamma+1,1}$ for some natural number $\gamma$ (or a convex polygon) and let $f \in L^{2}(\Omega)$. Then there exists $k_{0}$ large enough and $\delta>0$ small enough such that if $k \geq k_{0}, k h \leq \delta$ and $k^{p+1} h^{p} \leq \delta$ with $p \leq \gamma+1$ ( $p=1$ if $\Omega$ is a convex polygon), there exists a unique finite element solution $u_{h, p} \in V_{h, p}$ to (5.2), and the estimate

$$
\left\|| u - u _ { h , p } | | | \lesssim \operatorname { i n f } _ { \phi _ { h , p } \in V _ { h , p } } \left|\left\|u-\phi_{h, p} \mid\right\|\right.\right.
$$

holds. Furthermore, we have

$$
\left\|u-u_{h, p}\right\| \mid \lesssim k h\|f\|_{\Omega} .
$$

Proof. The proof of this Theorem is exactly the same as the one of Theorem 2 from [7], by using Theorem 4.2 and the fact that the sesquilinear form $b_{k}$ satisfies Assumption 1 from [7]. Indeed the continuity property

$$
\left|b_{k}(v, w)\right| \lesssim|||v||||||w|||, \forall v, w \in H_{0}^{1}(\Omega),
$$

is a direct consequence of Cauchy-Schwarz's inequality. Let us now prove the Gårding inequality

$$
\left.\operatorname{Re} b_{k}(u, u)|\gtrsim| u\right|_{1, \Omega} ^{2}-k^{2}\|u\|_{\Omega}^{2}, \quad \forall u \in H_{0}^{1}(\Omega) .
$$

Fix an arbitrary $u \in H_{0}^{1}(\Omega)$. First by the properties (A.13), (A.15) and (A.17), for $k$ large enough we have

$$
\left.\operatorname{Re} b_{k}(u, u)\left|\geq \frac{1}{2}\right| u\right|_{1, \Omega} ^{2}-2 k^{2}\|u\|_{\Omega}^{2}-\frac{C}{k} \int_{\Omega_{P M L}}|\nabla u||u| d x,
$$

for some $C>0$ independent of $k$. Cauchy-Schwarz's inequality and Young's inequality then lead to

This proves (5.5).

$$
\operatorname{Re} b_{k}(u, u) \geq \frac{1}{4}|u|_{1, \Omega}^{2}-\left(2 k^{2}+\frac{C^{2}}{k^{2}}\right)\|u\|_{\Omega}^{2} .
$$

5.1.2. Pre-asymptotic error estimate. In this part, we aim at giving a pre-asymptotic error estimate for the problem (2.5). As in [15], we use an appropriate elliptic projection, in order to obtain the existence of a solution $u_{h, p}$ to (5.2) under a weaker condition than in the asymptotic range.

First, we define:

$$
\begin{aligned}
L_{q}(u) & :=q^{2} \frac{\partial^{2} u}{\partial \rho^{2}}+\frac{1}{\rho^{2}} \frac{\partial^{2} u}{\partial \theta^{2}}+\frac{q^{2}}{\rho} \frac{\partial u}{\partial \rho}+q \frac{\partial q}{\partial \rho} \frac{\partial u}{\partial \rho} \\
& =\frac{q}{\rho} \frac{\partial}{\partial \rho}\left(q \rho \frac{\partial u}{\partial \rho}\right)+\frac{1}{\rho^{2}} \frac{\partial^{2} u}{\partial \theta^{2}} \\
& =\Delta u+\left(1-q^{2}\right) \frac{\partial^{2} u}{\partial \rho^{2}}+\left(1-q^{2}\right) \frac{1}{\rho} \frac{\partial u}{\partial \rho}+q \frac{\partial q}{\partial \rho} \frac{\partial u}{\partial \rho} .
\end{aligned}
$$


Then, we look at the following problem: find $u \in H_{0}^{1}(\Omega) \cap H^{2}(\Omega)$ solution of

$$
\begin{cases}L_{q}(u)=f & \text { in } \Omega, \\ u=0 & \text { on } \partial \Omega .\end{cases}
$$

The variational form of this problem is: Find $u \in H_{0}^{1}(\Omega)$ such that

$$
a_{k}(u, v)=(f, v)_{L^{2}(\Omega)} \quad \forall v \in H_{0}^{1}(\Omega),
$$

with $f \in L^{2}(\Omega)$ and

$$
\begin{aligned}
a_{k}(u, v) & :=\int_{\Omega}\left(q \frac{\partial u}{\partial \rho} \frac{\partial(q \bar{v})}{\partial \rho}+\frac{1}{\rho^{2}} \frac{\partial u}{\partial \theta} \frac{\partial \bar{v}}{\partial \theta}\right) d x \\
& =\int_{\Omega}\left(q^{2} \frac{\partial u}{\partial \rho} \frac{\partial \bar{v}}{\partial \rho}+\frac{1}{\rho^{2}} \frac{\partial u}{\partial \theta} \frac{\partial \bar{v}}{\partial \theta}+q \frac{\partial q}{\partial \rho} \frac{\partial u}{\partial \rho} \bar{v}\right) d x .
\end{aligned}
$$

Lemma 5.2. There exist a unique solution $u \in H_{0}^{1}(\Omega)$ to problem (5.6), further we have $u \in H^{2}(\Omega)$ with

$$
\|u\|_{2, \Omega} \lesssim\|f\|_{\Omega}
$$

Proof. We first prove that $a_{k}$ is continuous and coercive. Indeed one trivially has

$$
\left|a_{k}(u, v)\right| \lesssim\left(\left\|q^{2}\right\|_{\infty}+1\right)\|u\|_{1, \Omega}\|v\|_{1, \Omega}+\left\|q \frac{\partial q}{\partial \rho}\right\|_{\infty}\|\nabla u\|_{\Omega}\|v\|_{\Omega}, \forall u, v \in H_{0}^{1}(\Omega) .
$$

Hence, with Lemma A.2, A.3, we have the existence of a constant independent from $k$ such that

$$
\left|a_{k}(u, v)\right| \lesssim\|u\|_{1, \Omega}\|v\|_{1, \Omega}, \forall u, v \in H_{0}^{1}(\Omega) .
$$

On the other hand, if $k$ is large enough, we have

$$
\begin{aligned}
\operatorname{Re} a_{k}(u, u) & \geq \min (\operatorname{Re} q, 1)\|\nabla u\|_{\Omega}^{2}-\left\|q \frac{\partial q}{\partial \rho}\right\|_{\infty}\|\nabla u\|_{\Omega}\|v\|_{\Omega} \\
& \geq C_{1}\|\nabla u\|_{\Omega}^{2}-\frac{C_{2}}{k}\|u\|_{1, \Omega}^{2} \\
& \geq\left(C_{1}-\frac{C_{2}}{k}\right)\|u\|_{1, \Omega}^{2} \\
& \gtrsim\|u\|_{1, \Omega}^{2} .
\end{aligned}
$$

Then, since $a_{k}$ is continuous and coercive, by Lax-Milgram Lemma, we have the existence and uniqueness of a solution $u \in H_{0}^{1}(\Omega)$ to (5.6). The strong ellipticity of $L_{q}$ gives us the 
$H^{2}(\Omega)$ regularity of $u$. So, $u \in H^{2}(\Omega) \cap H_{0}^{1}(\Omega)$, and we have

$$
\begin{aligned}
\|u\|_{2, \Omega} & \lesssim\|\Delta u\|_{\Omega} \\
& \lesssim\left\|L_{q}(u)\right\|_{\Omega}+\left\|1-q^{2}\right\|_{\infty}\|u\|_{2, \Omega}+\left\|1-q^{2}\right\|_{\infty}\|u\|_{1, \Omega}+\left\|q \frac{\partial q}{\partial \rho}\right\|_{\infty}\|u\|_{1, \Omega} \\
& \lesssim\|f\|_{\Omega}+\frac{1}{k}\|u\|_{2, \Omega}+\|u\|_{1, \Omega},
\end{aligned}
$$

hence for $k$ large enough, we obtain (5.7).

Lemma 5.3. We define the projections $\mathcal{P}_{h, p} u \in V_{h, p}$ and $\mathcal{P}_{h, p}^{*} u \in V_{h, p}$ as unique solutions to

$$
\begin{array}{ccc}
a_{k}\left(\mathcal{P}_{h, p} u, v_{h, p}\right) & =a_{k}\left(u, v_{h, p}\right) & \forall v_{h, p} \in V_{h, p}, \\
a_{k}\left(v_{h}, \mathcal{P}_{h, p}^{*} u\right) & =a_{k}\left(v_{h}, u\right) & \forall v_{h, p} \in V_{h, p} .
\end{array}
$$

If $u_{\phi} \in H_{0}^{1}(\Omega)$ solves $b_{k}\left(u_{\phi}, v\right)=(\phi, v)$ for all $v \in H_{0}^{1}(\Omega)$ for some $\phi \in L^{2}(\Omega)$, then we have

$$
\left\|u_{\phi}-\mathcal{P}_{h, p}^{*} u_{\phi}\right\|_{\Omega} \lesssim\left(h^{2}+k^{p} h^{p+1}\right)\|\phi\|_{\Omega}
$$

and

$$
\left\|u_{\phi}-\mathcal{P}_{h, p}^{*} u_{\phi}\right\|_{1, \Omega} \lesssim\left(h+(k h)^{p}\right)\|\phi\|_{\Omega} .
$$

Proof. The existence and uniqueness of $\mathcal{P}_{h, p} u$ and of $\mathcal{P}_{h, p}^{*} u$ comes from the coercivity and continuity of $a_{k}$. We recall that, by Theorem 4.2 (with $\ell=p-1$ ), we have

$$
u_{\phi}=\sum_{j=0}^{p-2} k^{j} u_{\phi}^{(j)}+r_{\phi}
$$

with

$$
\begin{aligned}
\left\|u_{\phi}^{(j)}\right\|_{j+2, \Omega} & \lesssim\|\phi\|_{\Omega} \\
\left\|r_{\phi}\right\|_{p+1, \Omega} & \lesssim k^{p}\|\phi\|_{\Omega} .
\end{aligned}
$$

By Céa's lemma, we have

$$
\left\|u_{\phi}-\mathcal{P}_{h, p}^{*} u_{\phi}\right\|_{1, \Omega} \lesssim \inf _{v_{h, p} \in V_{h, p}}\left\|u_{\phi}-v_{h, p}\right\|_{1, \Omega} \lesssim\left\|u_{\phi}-\mathcal{I}_{h, p} u_{\phi}\right\|_{1, \Omega} .
$$

To estimate this right-hand side, we use (5.8) and (5.9) and (5.1) to obtain

$$
\begin{aligned}
\left\|u_{\phi}-\mathcal{I}_{h, p} u_{\phi}\right\|_{1, \Omega} & \lesssim \sum_{j=0}^{p-2} k^{j}\left\|u_{\phi}^{(j)}-\mathcal{I}_{h, p} u_{\phi}^{(j)}\right\|_{1, \Omega}+\left\|r_{\phi}-\mathcal{I}_{h, p} r_{\phi}\right\|_{1, \Omega} \\
& \lesssim \sum_{j=0}^{p-2} k^{j} h^{j+1}\left\|u_{\phi}^{(j)}\right\|_{j+2, \Omega}+h^{p}\left\|r_{\phi}\right\|_{p+1, \Omega} \\
& \lesssim h \sum_{j=0}^{p-2} k^{j} h^{j}\left\|u_{\phi}^{(j)}\right\|_{j+2, \Omega}+(k h)^{p}\|\phi\|_{\Omega} .
\end{aligned}
$$


This proves that

$$
\left\|u_{\phi}-\mathcal{I}_{h, p} u_{\phi}\right\|_{1, \Omega} \lesssim\left(h+(k h)^{p}\right)\|\phi\|_{\Omega},
$$

and hence

$$
\left\|u_{\phi}-\mathcal{P}_{h, p}^{*} u_{\phi}\right\|_{1, \Omega} \lesssim\left(h+(k h)^{p}\right)\|\phi\|_{\Omega},
$$

Similarly we can show that

$$
\left\|u_{\phi}-\mathcal{I}_{h, p} u_{\phi}\right\|_{\Omega} \lesssim h\left(h+(k h)^{p}\right)\|\phi\|_{\Omega} .
$$

This estimate cannot be used to bound the $L^{2}$-norm of $u_{\phi}-\mathcal{P}_{h, p}^{*} u_{\phi}$, hence we use an AubinNitsche trick. For this, we introduce $\xi \in H_{0}^{1}(\Omega)$ solution to $a_{k}(\xi, v)=\left(u_{\phi}-\mathcal{P}_{h, p}^{*} u_{\phi}, v\right)$, for all $v \in H_{0}^{1}(\Omega)$. The existence and uniqueness of $\xi$ follow from the properties of $a_{k}$ and we have

$$
\begin{aligned}
\left\|u_{\phi}-\mathcal{P}_{h, p}^{*} u_{\phi}\right\|_{\Omega}^{2} & =a_{k}\left(\xi, u_{\phi}-\mathcal{P}_{h, p}^{*} u_{\phi}\right) \\
& =a_{k}\left(\xi-\mathcal{P}_{h, p} \xi, u_{\phi}-\mathcal{P}_{h, p}^{*} u_{\phi}\right) \\
& \leq\left\|u_{\phi}-\mathcal{P}_{h, p}^{*} u_{\phi}\right\|_{1, \Omega}\left\|\xi-\mathcal{P}_{h, p} \xi\right\|_{1, \Omega} \\
& \lesssim\left(h+(k h)^{p}\right)\|\phi\|_{\Omega} h\left\|u_{\phi}-\mathcal{P}_{h, p}^{*} u_{\phi}\right\|_{\Omega} \\
& \lesssim\left(h^{2}+k^{p} h^{p+1}\right)\|\phi\|_{\Omega}\left\|u_{\phi}-\mathcal{P}_{h, p}^{*} u_{\phi}\right\|_{\Omega} .
\end{aligned}
$$

Now, since we have introduced the elliptic projection and its approximation properties in Lemma 5.3, we can follow [15] to produce a pre-asymptotic error estimate.

Theorem 5.4. Assume that $k^{p+2} h^{p+1}$ is small enough, then there exists a unique solution $u_{h, p} \in V_{h, p}$ of problem (5.2) and it holds

$$
\left\|u-u_{h, p}\right\| \mid \lesssim\left(k h+k^{2 p+1} h^{2 p}\right)\|f\|_{\Omega} .
$$

Proof. We use Aubin-Nitsche's trick, that is why we introduce $\xi \in H_{0}^{1}(\Omega)$, which verifies $b_{k}(v, \xi)=\left(v, u-u_{h}\right)$, for all $v \in H_{0}^{1}(\Omega)$. Hence we have, by the above lemma,

$$
\begin{aligned}
\left\|u-u_{h, p}\right\|_{\Omega}^{2} & =b_{k}\left(u-u_{h, p}, \xi\right)=b_{k}\left(u-u_{h, p}, \xi-\mathcal{P}_{h, p}^{*} \xi\right) \\
& =-k^{2}\left(\tilde{d}^{2}\left(u-u_{h, p}\right), \xi-\mathcal{P}_{h, p}^{*} \xi\right)+a_{k}\left(u-u_{h, p}, \xi-\mathcal{P}_{h, p}^{*} \xi\right) \\
& =-k^{2}\left(\tilde{d}^{2}\left(u-u_{h, p}\right), \xi-\mathcal{P}_{h, p}^{*} \xi\right)+a_{k}\left(u-\mathcal{I}_{h, p} u, \xi-\mathcal{P}_{h, p}^{*} \xi\right) \\
& \lesssim k^{2}\left\|u-u_{h, p}\right\|_{\Omega}\left\|\xi-\mathcal{P}_{h, p}^{*} \xi\right\|_{\Omega}+\left\|u-\mathcal{I}_{h, p} u\right\|_{1, \Omega}\left\|\xi-\mathcal{P}_{h, p}^{*} \xi\right\|_{1, \Omega} \\
& \lesssim\left((k h)^{2}+k^{p+2} h^{p+1}\right)\left\|u-u_{h, p}\right\|_{\Omega}^{2}+\left(h^{2}+(k h)^{2 p}\right)\|f\|_{\Omega}\left\|u-u_{h, p}\right\|_{\Omega} .
\end{aligned}
$$

Then, if $k^{p+2} h^{p+1}$ and $k h$ are small enough,

$$
\left\|u-u_{h, p}\right\|_{\Omega} \lesssim\left(h^{2}+(k h)^{2 p}\right)\|f\|_{\Omega} .
$$


This allows to estimate the energy norm of $u-u_{h, p}$ as follows:

$$
\begin{aligned}
\| u-u_{h, p}||^{2} & \lesssim k^{2}\left\|u-u_{h, p}\right\|_{\Omega}^{2}+\left|u-u_{h, p}\right|_{1, \Omega}^{2} \\
& \lesssim k^{2}\left\|u-u_{h, p}\right\|_{\Omega}^{2}+\left|a_{k}\left(u-u_{h, p}, u-u_{h, p}\right)\right| \\
& \lesssim k^{2}\left\|u-u_{h, p}\right\|_{\Omega}^{2}+\left|a_{k}\left(u-u_{h, p}, u-u_{h, p}\right)-k^{2}\left(\tilde{d}^{2}\left(u-u_{h, p}\right), u-u_{h, p}\right)\right| \\
& \lesssim k^{2}\left\|u-u_{h, p}\right\|_{\Omega}^{2}+\left|b_{k}\left(u-u_{h, p}, u-u_{h, p}\right)\right| \\
& \lesssim k^{2}\left\|u-u_{h, p}\right\|_{\Omega}^{2}+\left|b_{k}\left(u-u_{h, p}, u-\mathcal{I}_{h, p} u\right)\right| \\
& \lesssim k^{2}\left\|u-u_{h, p}\right\|_{\Omega}^{2}+\left|\left\|u-u_{h, p}\left|\left\|\cdot||\left|u-\mathcal{I}_{h, p} u\right|\right\| .\right.\right.\right.
\end{aligned}
$$

Young's inequality gives us

$$
\left\|\left|u-u_{h, p}\|\mid \lesssim k\| u-u_{h, p}\left\|_{\Omega}+\right\|\left\|u-\mathcal{I}_{h, p} u\right\| \| .\right.\right.
$$

By (5.13), (5.10) and (5.11), we deduce that

$$
\left\|u-u_{h, p}\right\|\left\|\lesssim\left(k\left(h^{2}+(k h)^{2 p}\right)+h+(k h)^{p}\right)\right\| f \|_{\Omega},
$$

which proves (5.12) as $k h^{2} \lesssim k h$ and $h+(k h)^{p} \lesssim k h$.

5.2. A multiscale approach. An alternative to high-order polynomials for achieving stability is the computation of subscale corrections in a multiscale fashion. The approach was first used for numerical homogenization problems [21] and later applied to Helmholtz problems by [26]. A Petrov-Galerkin variant of this approach is studied in [16], while [6] discusses the case of variable coefficients, which is closely related to the present case of a PML. In order to state the PML setting in the framework of [6], it is convenient to reformulate the original boundary-value problem (2.1) in Cartesian coordinates as follows

$$
-\nabla \cdot A \nabla u-k^{2} d \tilde{d} u=-d \tilde{d} f \quad \text { in } \Omega \quad \text { and } \quad u=0 \text { on } \partial \Omega .
$$

The resulting coefficient matrix $A$ has been provided by [9] and reads

$$
A(\rho, \theta)=\left(\begin{array}{ll}
q \cos ^{2} \theta+q^{-1} \sin ^{2} \theta & \left(q-q^{-1}\right) \cos \theta \sin \theta \\
\left(q-q^{-1}\right) \cos \theta \sin \theta & q \sin ^{2} \theta+q^{-1} \cos ^{2} \theta
\end{array}\right)
$$

where it is understood that $q=q(\rho)$. This problem is equivalent to (2.1) (and thereby to $(2.3)$ ) in the sense that they have the same unique solution $u$. The reason why the multiscale method is stated for this version of the equation it has the structure of a standard Helmholtz equation with a nontrivial diffusion coefficient. For this case, stability and error estimates have been formulated in $[6,16,26]$, and they immediately apply to the present situation. As the equations are equivalent on the PDE level, the stability results from Section 3 remain valid. The corresponding alternative variational formulation (equivalent to $(2.1)$ or $(2.3))$ reads: find $u \in H_{0}^{1}(\Omega)$ such that

$$
\mathcal{A}_{k}(u, v)=(\tilde{f}, v)_{L^{2}(\Omega)}
$$

where $\tilde{f}:=-d \tilde{d} f$ and the sesquilinear form $\mathcal{A}_{k}$ is defined by

$$
\mathcal{A}_{k}(v, w):=(A \nabla v, \nabla w)_{L^{2}(\Omega)}-k^{2}(d \tilde{d} u, v)_{L^{2}(\Omega)} \quad \text { for any } v, w \in H_{0}^{1}(\Omega) .
$$


With help of the results from Section 3 it can be shown that $\mathcal{A}_{k}$ satisfies the following inf-sup condition.

Lemma 5.5. The sesquilinear form $\mathcal{A}_{k}$ satisfies

$$
\gamma(k) \lesssim \inf _{v \in H_{0}^{1}(\Omega) \backslash\{0\}} \sup _{w \in H_{0}^{1}(\Omega) \backslash\{0\}} \frac{\operatorname{Re} \mathcal{A}_{k}(v, w)}{\|v\||\|w\||}
$$

where $\gamma(k)>0$ satisfies $\gamma(k)^{-1} \sim k$.

Proof. Let $v \in H_{0}^{1}(\Omega)$ be given. We follow the approach of [22] and denote by $z \in H_{0}^{1}(\Omega)$ the solution to the following dual problem

$$
\mathcal{A}_{k}(\eta, z)=2 k^{2}(\eta, v)_{L^{2}(\Omega)} \quad \text { for all } \eta \in H_{0}^{1}(\Omega) .
$$

The form $\mathcal{A}_{k}$ is symmetric (but not self-adjoint) and so the stability bound from Theorem 3.6 applies to $z$ and reads

$$
\left\|\left|\|z \mid\| \lesssim\left\|k^{2} v\right\|_{L^{2}(\Omega)} \lesssim k\|\| v\|\| .\right.\right.
$$

After setting $w:=v+z$ one concludes

$$
\mathcal{A}_{k}(v, w)=\mathcal{A}_{k}(v, v)+\mathcal{A}_{k}(v, z)=\mathcal{A}_{k}(v, v)+2 k^{2}\|v\|_{L^{2}(\Omega)}^{2}=\|\| v\|\|^{2}
$$

as well as

$$
|||w||| \leq|||v|||+|||z||| \lesssim(1+k)|||v||| \lesssim k|||v|||
$$

for $k$ large enough. The combination of these estimates yields

$$
\operatorname{Re} \mathcal{A}_{k}(v, w)=\left\||| v||^{2} \gtrsim k^{-1}|||v|\right\||\|w \mid\|
$$

which implies the claimed stability condition with $\gamma(k)^{-1} \lesssim k$.

Conversely, if we assume that (5.15) holds, then we have

$$
\gamma(k) \mid\|u\| \| \lesssim \sup _{w \in H_{0}^{1}(\Omega) \backslash\{0\}} \frac{\operatorname{Re} \mathcal{A}_{k}(u, w)}{\|w\| \mid}
$$

for the solution $u \in H_{0}^{1}(\Omega)$ of (5.14) with $f \in L^{2}(\Omega)$. Consequently by Cauchy-Schwarz's inequality one gets

$$
\gamma(k)\|\| u\|\| \lesssim \frac{\|f\|_{0, \Omega}}{k}
$$

or equivalently

$$
\|\mid u\|\left\|\frac{1}{k \gamma(k)}\right\| f \|_{0, \Omega} .
$$

According to the definition of $C_{\text {opt }}(k)$ from Lemma 3.2, we deduce that

$$
\frac{1}{k \gamma(k)} \gtrsim C_{\mathrm{opt}}(k)
$$

which proves the converse bound for $\gamma(k)^{-1}$ due to the equivalence (3.2). 
The numerical method is based on a coarse quasi-uniform finite element grid $\mathcal{T}_{H}$ and first-order conforming finite elements $V_{H, 1}$. The mesh size is indicated by the symbol $H$ because $h$ will refer to the fine-scale discretization parameter in the two-scale method. Let $\mathcal{J}_{H}: H_{0}^{1}(\Omega) \rightarrow V_{H, 1}$ denote a quasi-interpolation operator satisfying the usual first-order approximation and stability property

$$
H^{-1}\left\|v-\mathcal{J}_{H} v\right\|_{L^{2}(T)}+\left\|\nabla \mathcal{J}_{H} v\right\|_{L^{2}(T)} \lesssim\|\nabla v\|_{L^{2}(N(T))} \quad \text { for all } T \in \mathcal{T}_{H} \text { and all } v \in H_{0}^{1}(\Omega) \text {. }
$$

Here, $N(T)=N^{1}(T)$ is the union of all elements from $\mathcal{T}_{H}$ that have a nonempty intersection with $T$. More generally, we define $N^{0}(T):=T$ and

$$
N^{m}(T):=\bigcup\left\{K \in \mathcal{T}_{H}: K \cap N^{m-1}(T) \neq \emptyset\right\}
$$

for any positive integer $m$. On quasi-uniform meshes, the cardinality of $N^{m}(T)$ grows polynomially with $m$.

Let $h$ denote the fine-scale mesh parameter and consider the finite element space $V_{h, 1}$ related to the mesh $\mathcal{T}_{h}$. It is supposed that $\mathcal{T}_{h}$ is sufficiently fine such that the finite element method over $V_{h, 1}$ is stable in the sense that

$$
\gamma(k) \lesssim \inf _{v_{h} \in V_{h, 1} \backslash\{0\}} \sup _{w_{h} \in V_{h, 1} \backslash\{0\}} \frac{\operatorname{Re} \mathcal{A}_{k}\left(v_{h}, w_{h}\right)}{\left\|v _ { h } \left|\left\|||\left|w_{h}\right|\right\|\right.\right.}
$$

where $\gamma(k)$ is the inf-sup constant of $\mathcal{A}_{k}$ from Lemma 5.5. More precisely, if we assume that $k^{2} h$ is small enough, then (5.16) holds. Indeed let us introduce

$$
\eta\left(V_{h, 1}\right)=\sup _{f \in L^{2}(\Omega) \backslash\{0\}} \inf _{v_{h} \in V_{h, 1}} \frac{\left\|\mid \mathbb{S}_{k}^{*} f-v_{h}\right\|}{\|f\|_{0, \Omega}},
$$

where $\mathbb{S}_{k}^{*} f \in H_{0}^{1}(\Omega)$ is the solution of the adjoint problem of (5.14) with a right-hand side $f$. Then by standard interpolation estimates and the $H^{2}$ regularity of $\mathbb{S}_{k}^{*} f$, we can see that

$$
\eta\left(V_{h, 1}\right) \lesssim k h
$$

Consequently by using the arguments of [23, Thm 4.2] and the stability bound from Theorem 3.6, we deduce that (5.16) as soon as $k^{2} h$ is small enough.

Since global computations with $\mathcal{T}_{h}$ are too costly, only certain functions from $V_{h, 1}$ with quasi-local support will be utilized to stabilize a scheme over $\mathcal{T}_{H}$. The stabilization is as follows. The kernel of $\mathcal{J}_{H}$ reads

$$
W_{h}=\left\{v_{h} \in V_{h, 1}: \mathcal{J}_{H} v_{h}=0\right\} .
$$

Given $T \in \mathcal{T}_{H}$ and $v_{H} \in V_{H, 1}$, its so-called element correction $\mathcal{C}_{T} v_{h} \in W_{h}$ is defined as the solution to the following variational problem

$$
\mathcal{A}_{k}\left(w_{h}, \mathcal{C}_{T} v_{H}\right)=\mathcal{A}_{k, T}\left(w_{h}, v_{H}\right) \text { for all } w_{h} \in W_{h} .
$$

Here and throughout this section, the notation $\mathcal{A}_{k, \omega}$ indicates the spatial restriction of the form $\mathcal{A}_{k}$ to a subdomain $\omega$. Problem (5.17) is well-posed due to the next result. 
Lemma 5.6. Provided $H k \lesssim 1$, we have the coercivity

$$
\left\|\nabla w_{h}\right\|_{L^{2}(\Omega)}^{2} \lesssim \operatorname{Re} \mathcal{A}_{k}\left(w_{h}, w_{h}\right) \quad \text { for all } w_{h} \in W_{h} .
$$

The constants involved in " $\lesssim$ " only depend on the bounds of the coercivity and continuity constant of $A$ as well as on the maximal modulus of $d \tilde{d}$.

Proof. The proof almost verbatim follows [6, Lemma 1].

This result readily implies boundedness of $\mathcal{C}_{T}$,

$$
\left\|\left|\mathcal{C}_{T} v_{H}\|\| \lesssim\left\|\left|v_{H}\right|\right\|\right|_{T} \quad \text { for all } v_{H} \in V_{H} .\right.
$$

By linearity, one can see that the "global corrector" $\mathcal{C} v_{H}:=\sum_{T \in \mathcal{T}_{H}} \mathcal{C}_{T} v_{H}$ solves

$$
\mathcal{A}_{k}\left(w_{h}, \mathcal{C} v_{H}\right)=\mathcal{A}_{k}\left(w_{h}, v_{H}\right) \text { for all } w_{h} \in W_{h},
$$

and thus satisfies the continuity

$$
\left\|\left|\mathcal{C} v_{H}\right||| \lesssim\right\|\left|v_{H}\right| \| \text { for all } v_{H} \in V_{H} .
$$

As mentioned above, the correctors from (5.17) shall serve as an additive stabilizing component to the coarse finite element basis functions. But at this stage (5.17) defines a global fine-scale problem and, thus, $\mathcal{C}_{T} v_{H}$ is not computationally available. The key observation from [21] is that such computations can be localized to certain neighbourhoods of $T$. Let $\ell \in \mathbb{N}$ be a localization (or oversampling) parameter and define

$$
\Omega_{T}:=\operatorname{int} N^{\ell}(T)
$$

and

$$
W_{h}\left(\Omega_{T}\right):=\left\{w_{h} \in W_{h}: w_{h}=0 \text { outside } \Omega_{T}\right\} .
$$

These objects depend on the parameter $\ell$, which will, however, be suppressed for convenient notation. Problem (5.17) is now approximated by seeking $\mathcal{C}_{T, \ell} v_{H} \in W_{h}\left(\Omega_{T}\right)$ such that

$$
\mathcal{A}_{k, \Omega_{T}}\left(w_{h}, \mathcal{C}_{T, \ell} v_{H}\right)=\mathcal{A}_{k, T}\left(w_{h}, v_{H}\right) \quad \text { for all } w_{h} \in W_{h} .
$$

Note that the numerical computation of each of the problems (5.18) is feasible (with $\mathcal{O}(\ell H / h)^{2}$ vertices in 2D) as long as $\ell$ is of moderate size. The global localized version of $\mathcal{C}$ is defined as

$$
\mathcal{C}_{\ell} v_{H}:=\sum_{T \in \mathcal{T}_{H}} \mathcal{C}_{T, \ell} v_{H}
$$

The localized approximation is justified by the following exponential decay result.

Theorem 5.7. Provided $k H \lesssim 1$, there exists $0<\beta<1$ such that any $v_{H} \in V_{H}$, any $T \in \mathcal{T}_{H}$, and any $\ell \in \mathbb{N}$ satisfy

$$
\begin{aligned}
\left\|\nabla\left(\mathcal{C}_{T}-\mathcal{C}_{T, \ell}\right) v_{H}\right\|_{L^{2}(\Omega)} & \lesssim \beta^{\ell}\left\|\nabla v_{H}\right\|_{L^{2}(T)}, \\
\left\|\nabla\left(\mathcal{C}-\mathcal{C}_{\ell}\right) v_{H}\right\|_{L^{2}(\Omega)} & \lesssim C(\ell) \beta^{\ell}\left\|\nabla v_{H}\right\|_{L^{2}(T)},
\end{aligned}
$$

with a constant $C(\ell)$ that grows not faster than polynomially with $\ell$.

Proof. For a proof we refer to [16]. See also [6, Theorem 4]. 
The multiscale scheme is a Petrov-Galerkin method and referred to as multiscale PetrovGalerkin scheme (MSPG). It seeks $u_{H}^{(\ell)} \in V_{H, 1}$ such that

$$
\mathcal{A}_{k}\left(u_{H}^{(\ell)},\left(1-\mathcal{C}_{\ell}\right) v_{H}\right)=\left(\tilde{f},\left(1-\mathcal{C}_{\ell}\right) v_{H}\right)_{L^{2}(\Omega)} \quad \text { for all } v_{H} \in V_{H, 1} .
$$

Well-posedness of (5.19) is ensured through an appropriate parameter choice that will be described in the following. Suppose the fine-scale mesh size $h$ is small enough such that (5.16) is satisfied. The important property of the multiscale method is that it suffices to relate the oversampling lengths logarithmically to the wave number $k$.

Theorem 5.8. Suppose $k H \lesssim 1$ and (5.16) as well as

$$
\ell \gtrsim|\log \gamma(k)| /|\log \beta| \text {. }
$$

Then, the Petrov-Galerkin bilinear form from (5.19) satisfies

$$
\gamma(k) \lesssim \inf _{v_{H} \in V_{H, 1} \backslash\{0\}} \sup _{w_{H} \in V_{H, 1} \backslash\{0\}} \frac{\operatorname{Re} \mathcal{A}_{k}\left(v_{H},\left(1-\mathcal{C}_{\ell}\right) w_{H}\right)}{\||| v_{H}||||\left|w_{H}\right|||} .
$$

Proof. For a proof we refer to [16]. See also [6, Theorem 5].

As in $[16$, Thm 3], it can be shown that

$$
||\left|u_{h}-u_{H}^{(\ell)}\right|\left\|\left|\lesssim \inf _{v_{H} \in V_{H, 1}}\right|\left|u_{h}-v_{H}\right|\right\| .
$$

Thus, the triangle inequality and classical approximation properties together with the $H^{2}$ bound (3.5) show for $h$ sufficiently small that

$$
\|\| u-u_{H}^{(\ell)}\left|\|\mid \lesssim H\| u\left\|_{H^{2}(\Omega)} \lesssim H k\right\| f \|_{L^{2}(\Omega)} .\right.
$$

In particular, this means that the standard resolution condition $k H \lesssim 1$ for approximation is also sufficient for stability of the multiscale scheme.

\section{Some Numerical EXAMPLES}

6.1. A first example. For the first test, we have taken $\Omega=[-6,6]^{2} \backslash B(0,1)$, the fictious absorption coefficient $\sigma$ and the exact solution $u_{e x}$ as follows:

$$
\sigma(\rho)=\left\{\begin{array}{ll}
0 & \text { if } \rho \leq 4 \\
\frac{(\rho-4)^{2}}{2} & \text { otherwise }
\end{array} \quad \text { and } \quad u_{e x}(x, y)=\left(x^{2}-36\right)\left(y^{2}-36\right) e^{i k x} .\right.
$$

In Figure 2, we have depicted the rates of convergence for different values of $h$ and $k$, for $p=1$ and 2. We can see that, when $h$ is small enough, the order of convergence is $p$, as expected from the theory. From these plots we can observe three states of convergence: no convergence range/ pre-asymptotic range / asymptotic range.

Theorem 5.1 states that, provided $k^{p+1} h^{p} \lesssim 1$, the following error bound holds

$$
\left\|u_{e x}-u_{h, p}\left|\left\|\lesssim|| u_{e x}-P_{h, p} u_{e x}\right\|\right|,\right.
$$




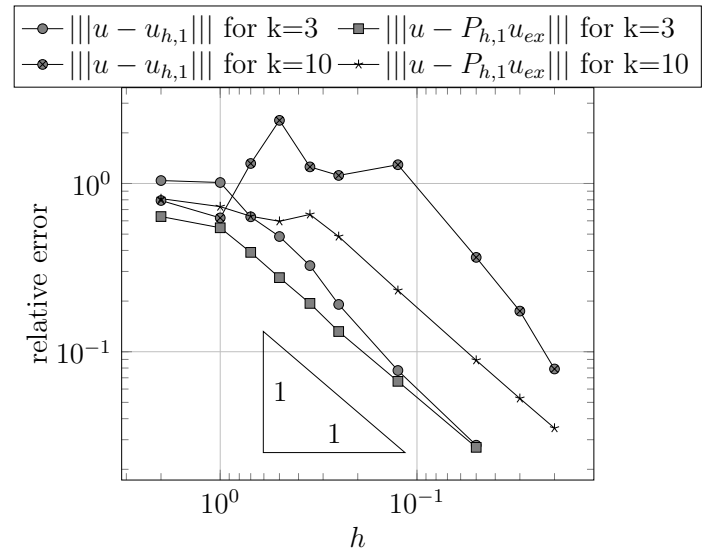

(a) Rates of convergence for $k=3$ or 10 with $p=1$

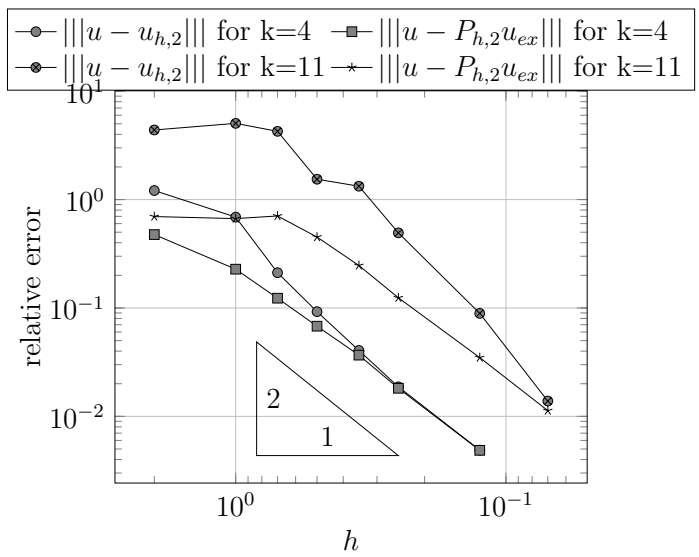

(b) Rates of convergence for $k=4$ or 11 with $p=2$

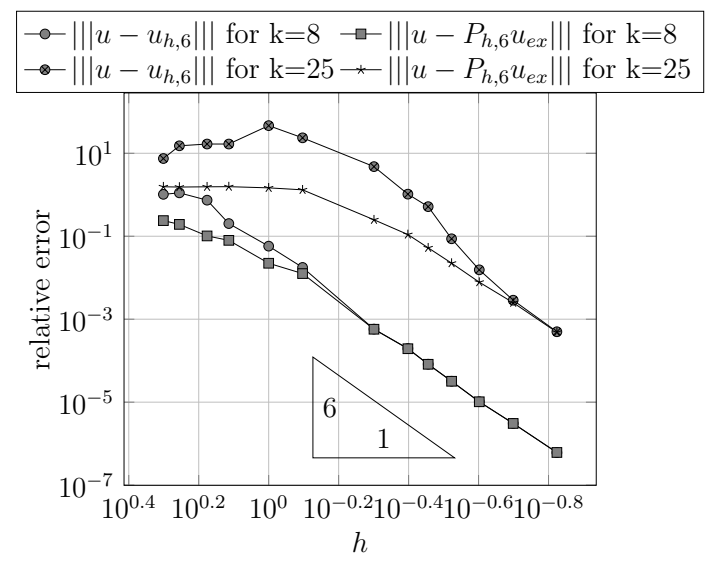

(c) Rates of convergence for $k=8$ or 25 with $p=6$

FiguRE 2. First experiment with $h p$ FEM: convergence curves for different values of $k$ and $p$.

where $P_{h, p} u_{e x}$ the orthogonal projection of $u_{e x}$ on $V_{h, p}$ for the inner product associated with the norm $\||\cdot|\| \mid$. For different values of $k, h$ and $p$, we compute $u_{h, p}$ and $P_{h, p} u_{e x}$, and denote by $h^{*}(k)$ the greatest value of $h$ such that

$$
||\left|u_{e x}-u_{h, p}\right|\left\|\leq 2||\left|u_{e x}-P_{h, p} u_{e x} \|\right| .\right.
$$

Figure 3 displays the graph of $h^{*}(k)$ (in a log-log scale) for $p=1$ and 2. In both cases, we observe that $h^{*}(k) \sim k^{-1-1 / p}$, which means that the condition $k^{p+1} h^{p} \lesssim 1$ is optimal. Figure 4 displays the relative errors in the preasymptotic range dependent on the wavenumber $k$, while $k$ and $h$ are coupled (depending on $p$ ) as in Theorem 5.4. As predicted by the theory, the relative error stays constant, which means that the discretization is stable with that choice of $h$ and $p$. 


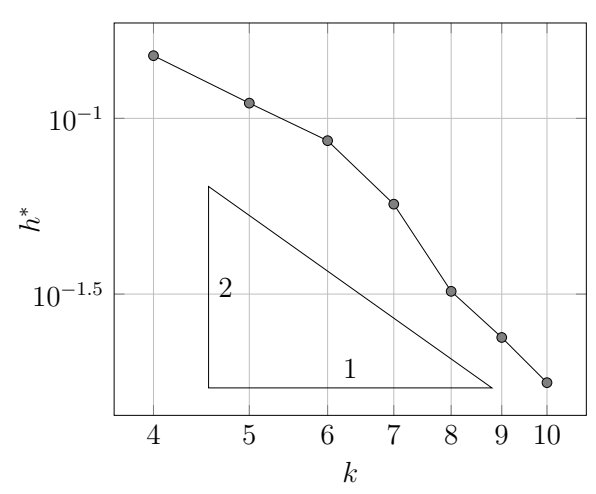

(a) $p=1$

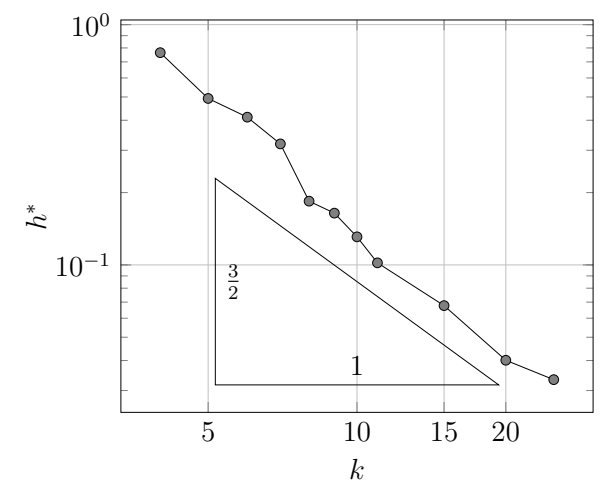

(b) $p=2$

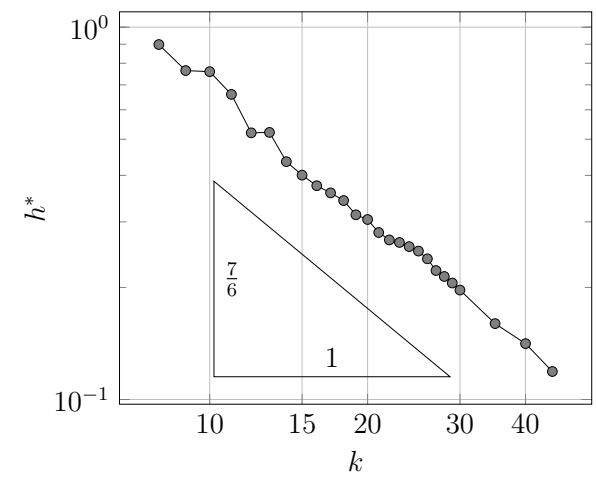

(c) $p=6$

Figure 3. First experiment with $h p$ FEM: Asymptotic range of $h^{*}(k)$ for $p=1,2$ and 6 .

Next, we report numerical results for the multiscale scheme. We consider $Q_{1}$ (bilinear) finite elements on a sequence of uniformly refined square meshes of mesh size $H=3 / 4,3 / 8, \ldots, 3 / 128$. The reference mesh has the mesh size $h=3 / 256$. The very regular structure of square meshes allows a quite efficient numerical implementation [16] of the method in which the correctors $\mathcal{C}_{\ell}$ outside the PML are computed on a reference patch and re-used where the same configuration occurs. For simplicity, we disregard the possibility of resolving the curved boundary within the corrector problems, although this can be done in principle $[13,27]$. We do not further analyze the error caused by this geometric perturbation. For wave numbers $k=8,12,16$, Figure 5 compares the relative errors in the energy norm $\|\cdot \cdot\| \mid$, namely the nodal interpolation by $Q_{1}$ finite element functions, the $Q_{1}$-FEM error, and the error of the MSPG method where the oversampling parameter varies from $\ell=1$ to $\ell=3$. For the FEM, pollution is clearly visible, while the MSPG scheme produces smaller errors that are close to the best approximation for appropriate $\ell$. Especially in the case $k=16$, the choice of $\ell=1$ seems to be insufficient, while $\ell=2,3$ lead to better results. This indicates the necessity of the coupling $\ell \sim \log k$. Since the accuracy of the MSPG method is limited by that of the FEM on the reference mesh, the 


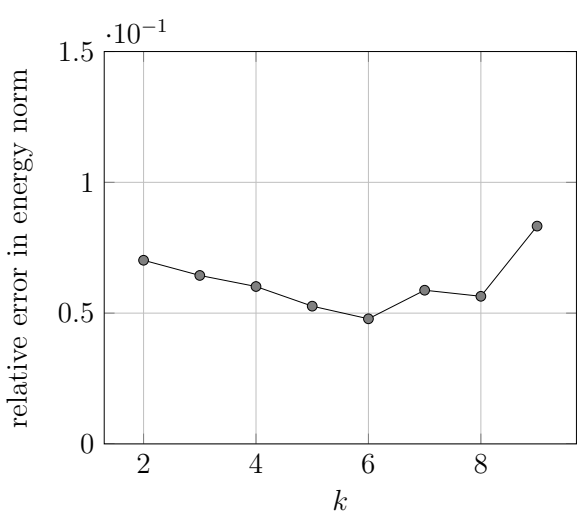

(a) $p=1$ with $k^{3} h^{2}$ constant

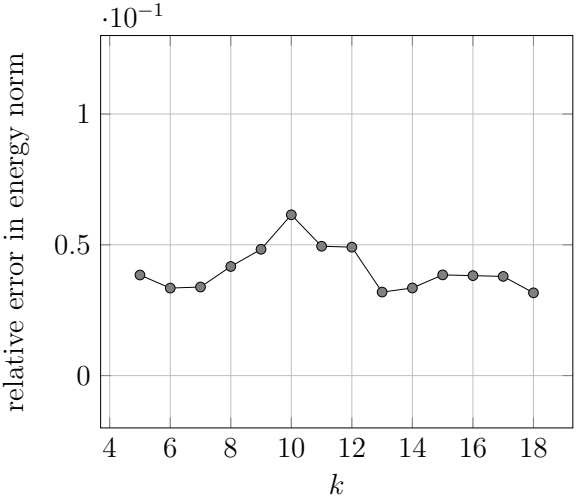

(b) $p=2$ and $k^{5} h^{4}=C$

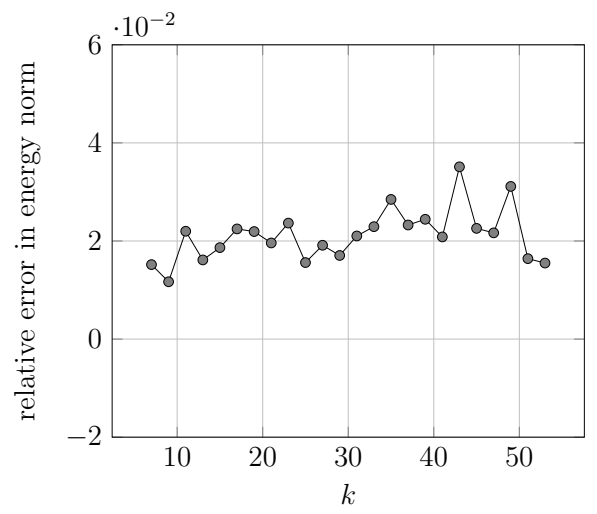

(c) $p=6$ and $k^{13} h^{12}=C$

FiguRE 4. First experiment with $h p$ FEM: preasymptotic range.

last two mesh refinements for $k=16$ do no provide a reasonable improvement. We finally mention that the mesh resolution condition " $h k^{2}$ small" is not fully satisfied for $k=16$, but we empirically observe that this choice of $h$ seems to be sufficient.

6.2. A scattering problem. Here we want to show the efficiency of our method by approaching a real scattering problem. Namely as obstacle $\mathcal{O}$ we take the unit disc and take

$$
u_{\text {scat }}(\theta, \rho)=\sum_{j=-\infty}^{\infty} i^{j}\left(\frac{J_{j}(k)}{J_{j}(k)+i Y_{j}(k)}\right)\left(J_{j}(k \rho)+i Y_{j}(k \rho)\right) e^{i j \theta}
$$

as exact solution of the Helmholtz equation in $\mathbb{R}^{2} \backslash \mathcal{O}$, which corresponds to the scattered solution of the incidence wave $e^{i k x_{1}}$ (see [20, (3.3)] or [10]). As fictitious absorption coefficient, we choose

$$
\sigma(\rho)=\left\{\begin{array}{ll}
0 & \text { if } \rho \leq a \\
\frac{\beta(\rho-a)^{2}}{(b-a)^{2}} & \text { otherwise }
\end{array},\right.
$$




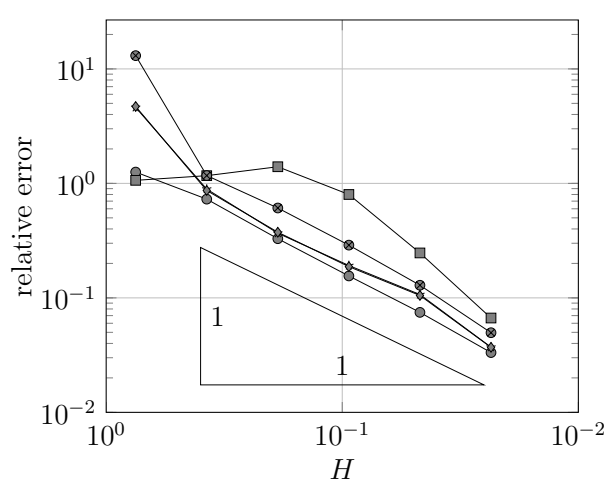

(a) $k=8$

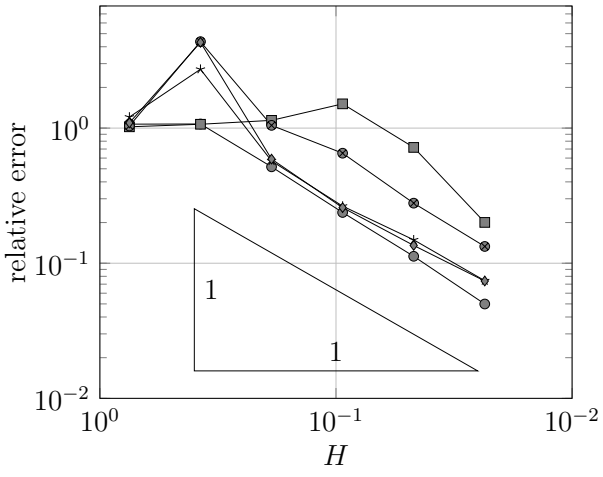

(b) $k=12$

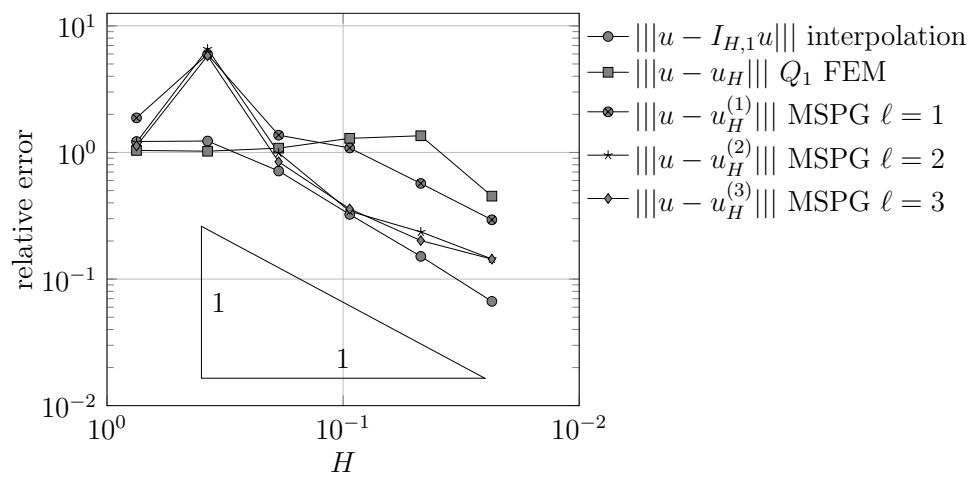

(c) $k=16$

FIgURE 5. First experiment. Relative error plots for the nodal interpolation $I_{H, 1} u$, the $Q_{1}$ FEM, and the multiscale Petrov-Galerkin method ('MSPG') with oversampling parameter $\ell=1,2,3$.

with $\beta>0$. Now, consider the solution $u_{b}$ of (compare with (2.3))

$$
\begin{cases}k^{2} \tilde{d}^{2} u_{b}+\frac{q}{\rho} \frac{\partial}{\partial \rho}\left(q \rho \frac{\partial u_{b}}{\partial \rho}\right)+\frac{1}{\rho^{2}} \frac{\partial^{2} u_{b}}{\partial \theta^{2}}=0 & \text { in } \Omega \\ u_{b}=e^{i k x_{1}} & \text { on } \partial \mathcal{O} \\ u_{b}=0 & \text { on } \partial \Omega \backslash \partial \mathcal{O}\end{cases}
$$

where $\Omega=B(0, b)$ (see section 2) with $1<a<b$. It is well-known (see for instance) $[18,19,5])$ that $u_{b}$ converges to $u_{\text {scat }}$ (even exponentially but the constant being dependent of the wave number $k)$ in $H^{1}(B(0, a))$ as $b$ goes to infinity. For our tests, we take $a=3$ and $b=6$.

As an approximation we compute

$$
u_{h, p} \in \tilde{V}_{h, p}=\left\{v_{h, p} \in H^{1}(\Omega)\left|v_{h, p}\right|_{K} \circ \mathcal{F}_{K}^{-1} \in \mathbb{P}_{p}(\hat{K}) \forall K \in \mathcal{T}_{h}\right\},
$$

the FEM solution of (6.1). 


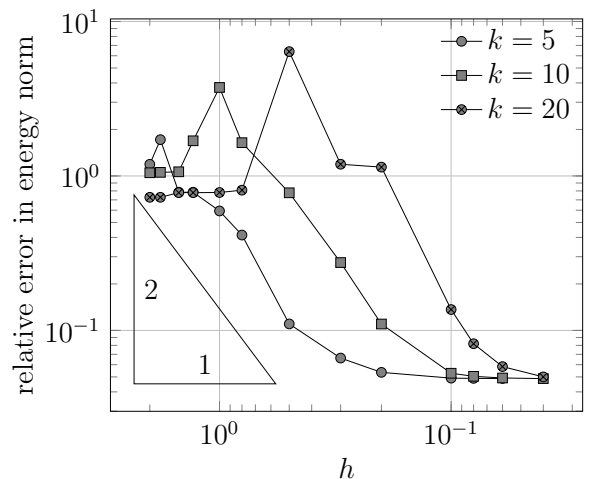

(a) $\beta=3$

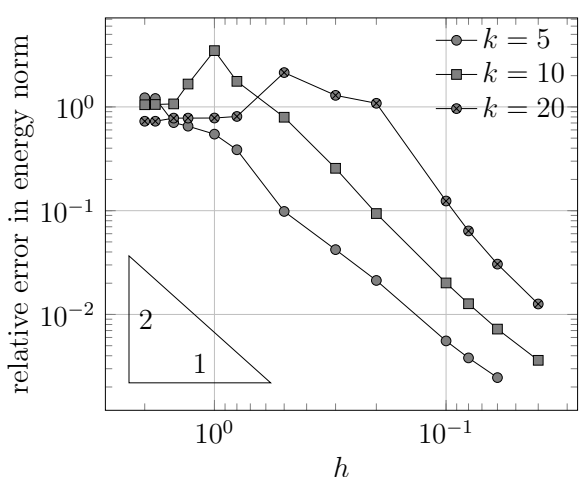

(b) $\beta=6$

Figure 6. Second experiment with $h p$ FEM: Convergence curves for different values of $k$ and $\beta$, with $p=2$.

As $u_{b}$ is unknown, we compare the FEM solution $u_{h, p}$ with $u_{\text {scat }}$, and the relative error in energy norm means that we compute $\frac{\|\left|u_{h, p}-u_{\text {scat }}\right| \mid \Omega_{\Omega_{a}}}{\|\left.|| u_{\text {scat }}||\right|_{\Omega_{a}}}$. The full error clearly satisfies

$$
||\left|u_{h, p}-u_{\text {scat }}\right|||_{\Omega_{a}} \leq||\left|u_{h, p}-u_{b}\right|||+\left.||\left|u_{b}-u_{\text {scat }}\right|\right|_{\Omega_{a}}
$$

Figure 6 shows convergence curves for different values of $k$, given in the relative energy norm by using polynomials of degree 2 . On the left, we have chosen $\beta=3$ small enough so that the error $\left\|u_{b}-u_{\text {scat }} \mid\right\|$ is not negligible. Accordingly, the error does not tend to 0 when $h$ is small. On the right, with $\beta=6$, the term $\left\|u_{b}-u_{\text {scat }}\right\|||$ is negligible compared to the FEM error. As $\sigma \in \mathcal{C}^{2}(\Omega)$, we know that $u_{b}$ is at least $H^{3}(\Omega)$, which is the reason why we have 2 for the convergence rate. Figure 7 shows for polynomials of degree 6 that the empirical convergence rate is not higher than 2.5 , which indicates that the solution $u_{b}$ might not be smoother than $H^{7 / 2}$. In comparison with the case $p=2$, in the case $\beta=6$, the term ||$\left|u_{b}-u_{\text {scat }}\right|||$ seems here more dominant as the rate of convergence deteriorates more rapidly.

We also made a pre-asymptotic test (see Figure 8) with $p=2$ and $\beta=3$ or 6 . We observe that when $k^{5} h^{4}$ is constant, the relative error in energy norm is constant too, which is in accordance with the estimate (6.2) since in the pre-asymptotic range the second term of the right-hand side is negligible, while the first one is constant due to Theorem 5.4.

Figure 9 displays the real part of $u_{\text {scat }}$ and $u_{h, p}$, for $k=20, p=6$ and $\beta=10$, where we see a good agreement between the exact solution and its approximation in $\Omega_{a}$.

The computational results obtained by the MSPG method are displayed in Figure 10. The parameters $H, h, \ell$, and $k$ are chosen as in the first experiment, and $\beta=10$. As in the first experiment, the FEM suffers from pollution, which is mitigated by the MSPG method. The precision increases with larger $\ell$. 


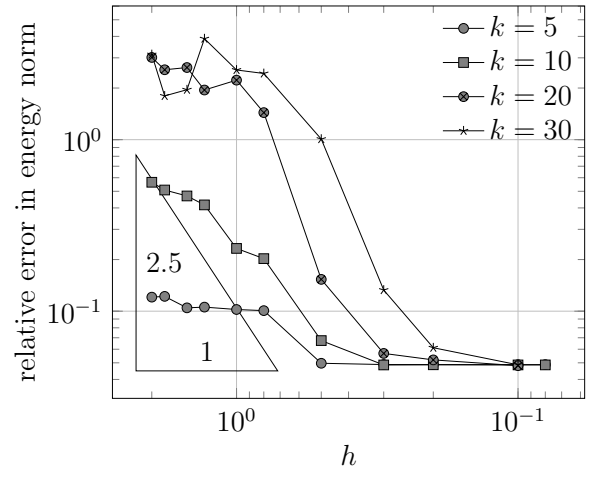

(a) $\beta=3$

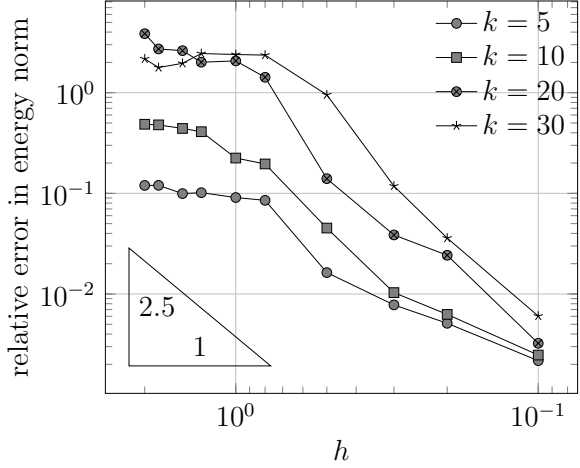

(b) $\beta=6$

Figure 7. Second experiment with $h p$ FEM: Convergence curves for different values of $k$ and $\beta$, with $p=6$.

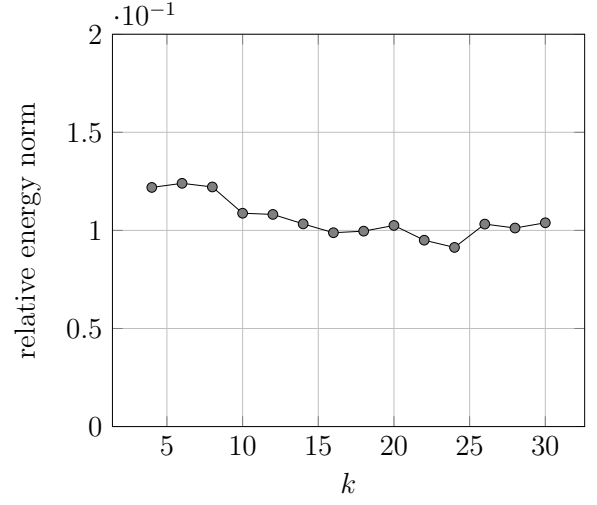

(a) $\beta=3$

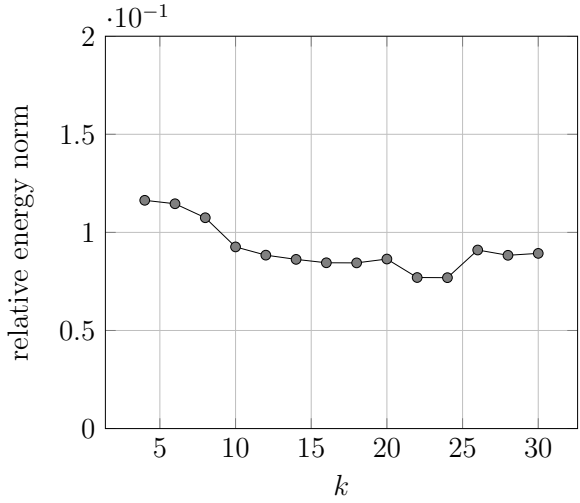

(b) $\beta=6$

FiguRE 8 . The pre-asymptotic examples with $p=2$.

\section{Conclusion}

We have shown that the PML model problem satisfies the $k$-stability property. This result enables is key to the numerical analysis of the two schemes presented in this work. The numerical results underline that the stability conditions for the numerical methods are sharp. Instead of comparing the two proposed schemes, we rather mention that they are are designed for different types of applications: the $h p$ FEM is of high order for smooth domains, while the multiscale scheme is pollution-free without smoothness, but restricted to first order. 

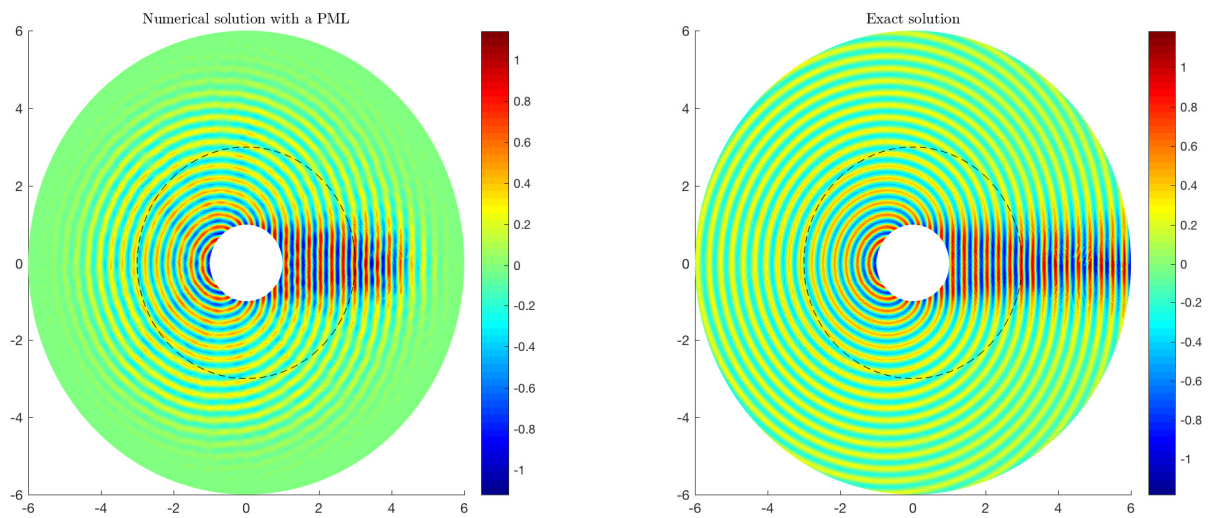

Figure 9. Second experiment with $h p$ FEM: Real part of the exact solution and the computed PML solution $(p=6$ and $\beta=10)$ with $k=20$.

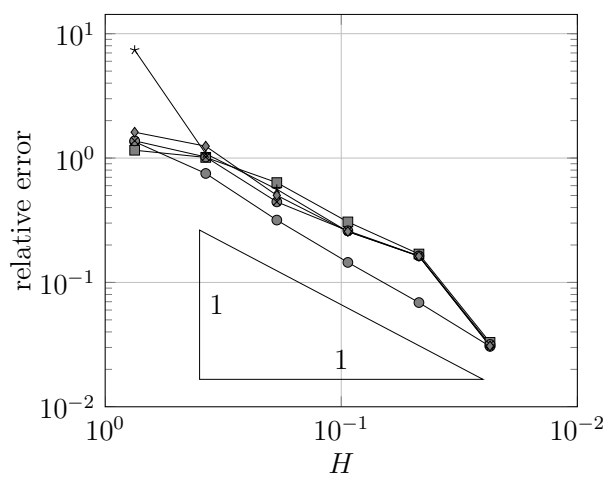

(a) $k=8$

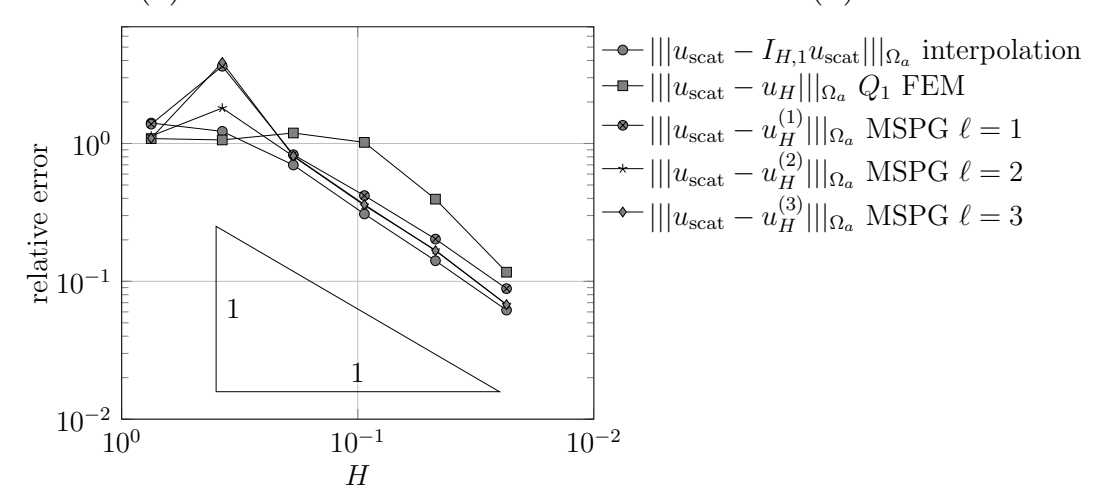

(c) $k=16$

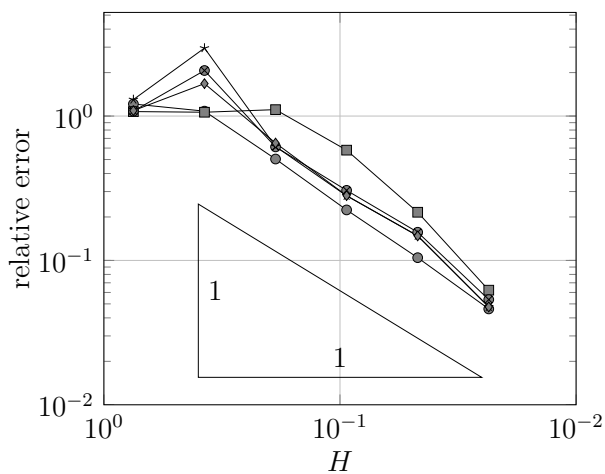

(b) $k=12$

FiguRE 10. Second experiment. Relative error plots for the nodal interpolation $I_{H, 1} u$, the $Q_{1}$ FEM, and the multiscale Petrov-Galerkin method ('MSPG') with oversampling parameter $\ell=1,2,3$. 


\section{REFERENCES}

[1] I. M. Babuška and S. A. Sauter. Is the pollution effect of the FEM avoidable for the Helmholtz equation considering high wave numbers? SIAM Rev., 42(3):451-484, 2000. Reprint of SIAM J. Numer. Anal. 34 (1997), no. 6, 2392-2423 [ MR1480387 (99b:65135)].

[2] J.-P. Berenger. A perfectly matched layer for the absorption of electromagnetic waves. J. Comput. Phys, 114:185-200, 1994.

[3] C. Bernardi. Optimal finite-element interpolation on curved domains. SIAM J. Numer. Anal., 26(5):1212-1240, 1989.

[4] T. Betcke, S. N. Chandler-Wilde, I. G. Graham, S. Langdon, and M. Lindner. Condition number estimates for combined potential integral operators in acoustics and their boundary element discretisation. Numer. Methods Partial Differential Equations, 27(1):31-69, 2011.

[5] J. H. Bramble and J. E. Pasciak. Analysis of a finite PML approximation for the three dimensional time-harmonic Maxwell and acoustic scattering problems. Math. Comp., 76(258):597-614, 2007.

[6] D. Brown, D. Gallistl, and D. Peterseim. Multiscale Petrov-Galerkin method for high-frequency heterogeneous Helmholtz equations. In M. Griebel and M. A. Schweitzer, editors, Meshfree Methods for Partial Differential Equations VII, volume 115 of Lect. Notes Comput. Sci. Eng., pages 85-115. 2017.

[7] T. Chaumont-Frelet and S. Nicaise. Wavenumber explicit convergence analysis for finite element discretizations of general wave propagation problems. Technical report, Lamav, Univ. Valenciennes, 2017.

[8] P. G. Ciarlet. The finite element method for elliptic problems. North-Holland, Amsterdam, 1978.

[9] F. Collino and P. Monk. The perfectly matched layer in curvilinear coordinates. SIAM J. Sci. Comput., 19(6):2061-2090, 1998.

[10] D. Colton and R. Kress. Inverse acoustic and electromagnetic scattering theory, volume 93 of Applied Mathematical Sciences. Springer-Verlag, Berlin, second edition, 1998.

[11] D. Colton and R. Kress. Inverse Acoustic and Electromagnetic Scattering Theory. Springer, 2012.

[12] P. Cummings and X. Feng. Sharp regularity coefficient estimates for complex-valued acoustic and elastic Helmholtz equations. Mathematical Models and Methods in Applied Sciences, 16(01):139-160, 2006.

[13] D. Elfverson, M. G. Larson, and A. Må lqvist. Multiscale methods for problems with complex geometry. Comput. Methods Appl. Mech. Engrg., 321:103-123, 2017.

[14] S. Esterhazy and J. Melenk. On stability of discretizations of the Helmholtz equation. In Lecture Notes in Computational Science and Engineering, pages 285-324. Springer-Verlag Berlin Heidelberg, 2012.

[15] X. Feng and H. Wu. hp-discontinuous Galerkin methods for the Helmholtz equation with large wave number. Mathematics of Computation, 80:1997-2024, 2011.

[16] D. Gallistl and D. Peterseim. Stable multiscale Petrov-Galerkin finite element method for high frequency acoustic scattering. Comput. Methods Appl. Mech. Eng., 295:1-17, 2015.

[17] U. Hetmaniuk. Stability estimates for a class of Helmholtz problems. Commun. Math. Sci., 5(3):665$678,2007$.

[18] M. Lassas and E. Somersalo. On the existence and convergence of the solution of PML equations. Computing, 60(3):229-241, 1998.

[19] M. Lassas and E. Somersalo. Analysis of the PML equations in general convex geometry. Proc. Roy. Soc. Edinburgh Sect. A, 131(5):1183-1207, 2001.

[20] H. Liu and J. Zou. Zeros of the Bessel and spherical Bessel functions and their applications for uniqueness in inverse acoustic obstacle scattering. IMA J. Appl. Math., 72(6):817-831, 2007.

[21] A. Målqvist and D. Peterseim. Localization of elliptic multiscale problems. Math. Comp., 83(290):2583-2603, 2014.

[22] J. Melenk. On generalized finite element methods. PhD thesis, University of Maryland, 1995. 
[23] J. Melenk and S. Sauter. Convergence analysis for finite element discretizations of the Helmholtz equation with Dirichlet-to-Neumann boundary conditions. Mathematics of Computation, 79(272):18711914, 2010.

[24] J. Melenk and S. Sauter. Wavenumber explicit convergence analysis for Galerkin discretizations of the Helmholtz equation. SIAM J. Numer. Anal., 49(3):1210-1243, 2011.

[25] J. M. Melenk. Mapping properties of combined field Helmholtz boundary integral operators. SIAM Journal on Mathematical Analysis, 44(4):2599-2636, 2012.

[26] D. Peterseim. Eliminating the pollution effect in Helmholtz problems by local subscale correction. Math. Comp., 86:1005-1036, 2017.

[27] D. G. Schissler. Multiscale Methods for Acousting Scattering. Master thesis, Universität Bonn, 2016.

[28] E. A. Spence. Wavenumber-explicit bounds in time-harmonic acoustic scattering. SIAM J. Math. Anal., 46(4):2987-3024, 2014.

\section{Appendix A. Useful properties of the PML Functions}

We recall from [9] that the fictious absorption coefficient $\sigma$ is supposed to be a non decreasing function in $C^{1}(0, \infty)$ such that

$$
\sigma(\rho)=\left\{\begin{array}{l}
\quad=0, \forall \rho \leq a \\
>0, \forall \rho>a .
\end{array}\right.
$$

Then we define $\tilde{\sigma} \in C[0, \infty)$ as follows

$$
\tilde{\sigma}(\rho)=\left\{\begin{array}{c}
=0, \forall \rho \leq a, \\
\frac{1}{\rho} \int_{a}^{\rho} \sigma(s) d s, \forall \rho>a .
\end{array}\right.
$$

From this expression, we deduce that

$$
\rho \tilde{\sigma}(\rho)=\int_{a}^{\rho} \sigma(s) d s, \forall \rho>a
$$

and therefore

$$
\sigma(\rho)=(\rho \tilde{\sigma})^{\prime}(\rho), \forall \rho>a
$$

By Leibniz's rule, we get

$$
\rho \tilde{\sigma}^{\prime}(\rho)=\sigma(\rho)-\tilde{\sigma}(\rho), \forall \rho>a .
$$

In addition, as $\sigma$ is non decreasing, (A.2) directly implies

$$
\tilde{\sigma}(\rho) \leq \frac{\rho-a}{\rho} \sigma(\rho)<\sigma(\rho), \forall \rho>a .
$$

These two estimates directly lead to

$$
\tilde{\sigma}^{\prime}(\rho)>0, \forall \rho>a,
$$

and therefore $\tilde{\sigma}$ is a also a non decreasing function. Furthermore $\tilde{\sigma} \in C^{1}[0, \infty)$ because from (A.3) and the continuity at $a$ of $\sigma$ and $\tilde{\sigma}$, one has

$$
\tilde{\sigma}^{\prime}(\rho)=\frac{\sigma(\rho)-\tilde{\sigma}(\rho)}{\rho} \rightarrow 0, \text { as } \rho \rightarrow a .
$$


From (A.1), (A.2) and (A.5) and the $C^{1}$ property of $\tilde{\sigma}$, for all $\varepsilon>0$, there exists $\delta>0$ such that

$$
\sigma(\rho) \geq \delta, \tilde{\sigma}(\rho) \geq \delta, \tilde{\sigma}^{\prime}(\rho) \geq \delta, \forall \rho \geq a+\frac{\varepsilon}{2} .
$$

We then fix $\varepsilon>0$ small enough such that $a+\varepsilon<b$ and fix a cut-off function $\eta \in \mathcal{D}(\mathbb{R})$ with $\eta^{\prime} \leq 0$ such that

$$
\eta(\rho)=\left\{\begin{array}{l}
1, \forall \rho \leq a+\frac{\varepsilon}{2} \\
0, \forall \rho \geq a+\varepsilon
\end{array}\right.
$$

For convenience, we denote by $\Omega_{P M L}$, the PML region, i. e.,

$$
\Omega_{P M L}=\{x \in \Omega:|x|>a\} .
$$

We also set

$$
\Omega_{P M L}^{+}=\left\{x \in \Omega:|x|>a+\frac{\varepsilon}{2}\right\} .
$$

Lemma A.1. We always have

$$
\sigma \leq \tilde{\sigma}^{\prime} \text { in } \Omega_{P M L}
$$

Proof. By (A.4), one has

$$
0 \leq \lim _{\rho \rightarrow a+} \frac{\tilde{\sigma}(\rho)}{\sigma(\rho)} \leq \lim _{\rho \rightarrow a+} \frac{\rho-a}{\rho}=0
$$

which shows that

$$
\lim _{\rho \rightarrow a+} \frac{\tilde{\sigma}(\rho)}{\sigma(\rho)}=0
$$

Using (A.3), we then have

$$
\lim _{\rho \rightarrow a+} \frac{\tilde{\sigma}^{\prime}(\rho)}{\sigma(\rho)}=\frac{1}{a} .
$$

Consequently for $\rho>a$ but close to $a$, we trivially have (A.7). On the other hand, for $\rho \in\left[a+\varepsilon_{1}, b\right]$, with $\varepsilon_{1}>0$ as small as we want, (A.6) and the continuity of $\sigma$ directly yield (A.7). The proof is then complete.

As in [9], we set

$$
d=1+\frac{i \sigma}{k}, \quad \text { and } \tilde{d}=1+\frac{i \tilde{\sigma}}{k} .
$$

Let us also define

$$
q=\frac{\tilde{d}}{d}
$$


Lemma A.2. The next properties hold

$$
\begin{array}{r}
\operatorname{Im} q(\rho)=-\frac{k \rho \tilde{\sigma}^{\prime}(\rho)}{k^{2}+\sigma^{2}(\rho)} \leq 0, \\
\operatorname{Im} q^{2}=2 \gamma \operatorname{Im} q, \\
\operatorname{Im} \tilde{d}^{2}=\frac{2 \tilde{\sigma}}{k} \geq 0, \\
q \rightarrow 1, \text { as } k \rightarrow \infty, \\
d \rightarrow 1, \text { as } k \rightarrow \infty, \\
\tilde{d} \rightarrow 1, \text { as } k \rightarrow \infty, \\
\quad \tilde{d}^{2}-\tilde{d}^{2}=-\frac{4 i}{k} \tilde{\sigma}
\end{array}
$$

where $0<\gamma=\frac{1+\frac{\sigma \tilde{\sigma}}{k^{2}}}{1+\frac{\sigma^{2}}{k^{2}}}$ that tends to 1 as $k$ goes to infinity.

Proof. The properties (A.12) to (A.16) are direct. To prove (A.10) and (A.11), we notice that $q$ admits the writing

$$
q=\gamma+\frac{i k}{k^{2}+\sigma^{2}}(\tilde{\sigma}-\sigma),
$$

which directly yields the results recalling (A.3).

Lemma A.3. We have

$$
\begin{array}{r}
\left|\frac{\partial}{\partial \rho} q\right| \lesssim \frac{1}{k} \text { in } \Omega_{P M L}, \\
q=1 \text { in } \Omega_{0}, \\
|q-1| \lesssim \frac{1}{k} \text { in } \Omega_{P M L} .
\end{array}
$$

Proof. The second identity being immediate, let us concentrate on the two other ones. By direct calculations, we see that

$$
\frac{\partial}{\partial \rho} q=\frac{i}{k}\left(\frac{\tilde{\sigma}^{\prime}}{d}-\frac{\tilde{d} \sigma^{\prime}}{d^{2}}\right) .
$$

The estimate (A.17) follows as $|d| \geq 1$ as well as $|\tilde{d}| \geq 1$ and since $\sigma^{\prime}$ and $\tilde{\sigma}^{\prime}$ are bounded.

Concerning the last one, we see that

$$
q-1=\frac{1}{k} \frac{i(\tilde{\sigma}-\sigma)}{1+\frac{i \sigma}{k}} .
$$

Hence the estimate (A.19) holds because $\left|1+\frac{i \sigma}{k}\right| \geq 1$ and because $\sigma$ and $\tilde{\sigma}$ are bounded. 
T. Chaumont-Frelet, Cermics, École des Ponts Paristech, Champs sur Marne, France E-mail address: theophile.chaumont@enpc.fr

D. Gallistl, Department of Applied Mathematics, University of Twente, P.O. Box 217 , 7500 AE Enschede, The Netherlands

E-mail address: d.gallistl@utwente.nl

S. Nicaise, Univ. Valenciennes, EA 4015 - LAMAV - FR CNRS 2956, F-59313 Valenciennes, France

E-mail address: Serge.Nicaise@uphf.fr

J. Tomezyk, Univ. Valenciennes, EA 4015 - LAMAV - FR CNRS 2956, F-59313 ValenciEnnes, France

E-mail address: Jerome.Tomezyk@uphf.fr 